Publ. RIMS, Kyoto Univ.

15 (1979), 797-826

\title{
Fixed Points of the Actions on Compact Kähler Manifolds
}

$\mathrm{By}$

Akira FuJIKI*,1)

\section{lnurrode}

Let $X$ be a compact Kähler manifold and $V$ a Killing vector field on $X$ with respect to a Kähler metric on $X$. Let $Z$ be the zero set of $V$ which is a complex submanifold of $X$ (cf. [12]). Suppose that $Z$ is nonempty and let $Z_{a}, \alpha=1, \cdots, m$, be the connected components of $Z$. Then in [8] Frankel showed the following: one can associate to each $\alpha$ naturally a nonnegative integer $\lambda_{a}$ such that $b_{i}(X)=\sum_{\alpha=1}^{m} b_{i-2 \lambda_{\alpha}}\left(Z_{\alpha}\right)$ for all $i \geqq 0$, where $b_{i}(Y)$ denotes the $i$-th Betti number of $Y$. In particular if $\chi$ denotes the Euler characteristic, then we have $\chi(X)=$ $\sum_{a} \chi\left(Z_{\alpha}\right)$. The main purpose of this paper is to sharpen this result in the following form.

Theorem. Let $X, V$ and $Z=\cup_{\alpha} Z_{\alpha}$ be as above. Then we have the equalities

$$
h^{p, q}(X)=\sum_{\alpha=1}^{m} h^{p-\lambda_{\alpha}, q-l_{\alpha}}\left(Z_{\alpha}\right), \quad p, q \geqq 0
$$

where $\lambda_{\alpha}$ are as above and $h^{p, q}(Y)=\operatorname{dim} H^{q}\left(Y, \Omega_{Y}^{p}\right)$.

Corrollary (Kosniowski [13]). Let $\chi^{p}(Y)=\sum_{q=0}^{\infty}(-1)^{q} h^{p, q}(Y)$ and $\chi_{y}(Y)=\sum_{y=0}^{\infty} \chi^{p}(Y) y^{p}$, where $y$ is an indeterminate. Then we have

$$
\chi_{y}(X)=\sum_{\alpha=1}^{m}(-1)^{\lambda_{\alpha}} \chi_{y}\left(Z_{\alpha}\right) .
$$

Received February 20, 1978.

* Research Institute for Mathematical Sciences, Kyoto University

1) Supported by the Sakkokai Foundation. 
Note that the result of Kosniowski is more general in that it applies also to general compact complex manifolds for a certain class of $V$. For a precise description of $\lambda_{\alpha}$ in the above theorem and corollary, see (3.5).

The organization of this paper is as follows. First in Section 1 we reduce the problem in the usual way to that of fixed points of an action of an algebraic torus $\left(\mathbb{C}^{*}\right)^{k}$ on $X$, as well as obtain some lemmas which are needed in the sequel. Then in Section 2 we consider the case $k=1$, and following Bialynicki-Birula [1] we study the decomposition, $X=\cup_{\alpha} X_{\alpha}$, of $X$ into a finite number of $\mathbb{C}^{*}$-invariant locally closed submanifolds $X_{\alpha}$. (This takes the place of Frankel's Morse theoretic argument.) The point here is that $X_{\alpha}$ are locally closed in the Zariski topology of $X$ (Theorem 2.2), which is due to Bialynicki-Birula when $X$ is projective. In fact, this enables us to apply the Hodge theory developped by Deligne in [7] to our problem in Section 3. Combining the result thus obtained with the inequality of Conner (Corollary 1.7) we then prove Theorem. Since what we need from Hodge theory (Lemmas 3.1, 3.2) does not seem explicit in the literature, we give proofs to these in a separate note [10].

In this paper all the complex spaces are assumed to be reduced.

\section{§. Some Lemmas}

Let $X$ be complex space and $G$ a connected complex Lie group acting biholomorphically on $X$. Thus we have a morphism $\sigma: G \times X \rightarrow X$ with $\sigma\left(g_{1}, \sigma\left(g_{2}, x\right)\right)=\sigma\left(g_{1} g_{2}, x\right), g_{i} \in G, x \in X$ and $\sigma(e, x)=x$, $e$ being the identity of $G$. We often write $g x$ for $\sigma(g, x)$. The set of fixed points, or the fixed point set, of $G$ on $X$ is the set $F=\{x \in X ; g x=x$ for all $g$ $\in G\}$. This is an analytic subset of $X$. Let $x \in F$ be a fixed point and $T_{x}$ the Zariski tangent space of $X$ at $x$. Let $\rho_{x}: G \rightarrow G L\left(T_{x}\right)$ be the isotropy representation of $G$ on $T_{x}$, where $G L\left(T_{x}\right)$ is the group of all invertible linear transformations of $T_{x}$. The following lemma and the ensuing corollary are well known.

Lemma 1. 1. Suppose that $G$ is reductive. Then there exists a neighborhood $U$ of $x$ in $X$ (resp. $Y$ of the origin in $T_{x}$ ) and $a G$ equivariant embedding $\psi: U \rightarrow V$ (i.e. for every pair $(g, x) \in G \times U$ with 
$g x \in U$, we get that $\left.\psi(g x)=\rho_{x}(g) \psi(x)\right)$.

Proof. Let $K$ be any maximal compact subgroup of $G$. Then there is a $K$-equivariant embedding $\psi: U \rightarrow V$ with $U$ and $V$ as in the lemma (cf. Kaup [11, Satz 4.4]). Since $G$ is the complexification of $K, \psi$ must also be $G$-equivariant in the sense mentioned above.

Q.E.D.

Corollary 1.2. If $X$ is nonsingular, then $F$ is a complex submanifold of $X$.

Proof. Take an arbitrary point $x \in F$. It is enough to show that for the neighborhood $U$ of $x$ in the above lemma $U \cap F$ is nonsingular. Then the problem is reduced to the case where $U$ is a neighborhood of the origin of a vector space $V$ on which $G$ acts linearly. Let $V_{0}$ be the maximal subspace of $V$ on which $G$ acts trivially. Then since $G$ is reductive, we can find a $G$-invariant subspace $V_{1}$ of $V$ with a $G$-isomorphism $V \cong V_{1} \oplus V_{0}$. Then it is clear that $U \cap F=U \cap V_{0}$ and hence is nonsingular.

Q.E.D.

We consider the case $G=\mathbb{C}^{*}$ in Lemma 1. 1 . Then $\rho_{x}$ is a direct sum of 1 -dimensional representations i.e. the characters $\chi_{1}, \cdots, \chi_{n}$, of $\mathbb{C}^{*}$, where $n=\operatorname{dim} T_{x}$. Let $\chi_{i}(t)=t^{m_{i}}, m_{i} \in \mathbb{Z}$, with $\mathbb{C}^{*}=\mathbb{C}^{*}(t)$. Then with respect to suitable linear coordinates $z_{1}, \cdots, z_{n}$ of $T_{x}$ the action $\sigma_{x}=\rho_{x}$ of $G$ on $T_{x}$ looks as follows;

$$
\sigma_{x}\left(t,\left(z_{1}, \cdots, z_{n}\right)\right)=\left(t^{m_{1}} z_{1}, \cdots, t^{m_{n}} z_{n}\right) .
$$

We shall identify $U$ with a subspace of $V$ by $\psi$ so that the action on $U$ is induced from (1) by the inclusion. Then $F \cap U$ is the intersection of $U$ with the linear subspace defined by the equations

$$
z_{i_{1}}=\cdots=z_{i_{s}}=0 \text {, }
$$

where $i \in\left\{i_{1}, \cdots, i_{s}\right\}$ if and only if $m_{i} \neq 0$. Note that the set $M_{x}=\left\{m_{1}\right.$, $\left.\cdots, m_{n}\right\}$ is independent of the particular choice of the coordinates, being an invariant of $\rho_{x}$.

Now in (1) let $I=\{1, \cdots, n\}$ and $I_{+}$(resp. $\left.I_{-}, I_{0}\right)=\left\{1 \leqq i \leqq n ; m_{i}>0\right.$ (resp. $<0,=0)\}$. We call the action positive (resp. negative) at $x$ if 
$I=I_{+}$(resp. $\left.I_{-}\right)$and semipositive (resp. seminegative) if $I_{-}$(resp. $I_{+}$) $=\varnothing$. We define $T_{-}=T_{+}(x) \quad$ (resp. $\left.T_{-}=T_{-}(x), T_{0}=T_{0}(x)\right)$ by $\left\{\left(z_{i}\right)\right.$; $z_{i}=0, i \notin I_{+} \quad\left(\right.$ resp. $\left.\left.I_{-}, I_{0}\right)\right\}$.

Lemma 1.3. 1) If the action is not semipositive (resp. seminegative) at $x$, then $\operatorname{dim}_{x} X \cap T_{-}$(resp. $\left.\operatorname{dim}_{x} X \cap T_{+}\right)>0$.

2) If the action is semipositive (resp. seminegative) and not positive (resp. negative) at $x$, then $\operatorname{dim}_{x} F>0$.

Proof. 1) Suppose that the action is not semipositive at $x$ i.e. $T_{\text {- }}$ $\neq\{0\}$. If $\operatorname{dim}_{x} X \cap T_{-}=0$, then the natural $\mathbb{C}^{*}$ equivariant projection $\pi: T_{x} \rightarrow T_{+} \oplus T_{0}$ with kernel $T_{-}$is finite at $x$ when restricted to $X$. From this we infer readily that the action is semipositive at $x$, which is a contradiction. Hence $\operatorname{dim}_{x} X \cap T_{-}>0$. The non-seminegative case can be treated in the same way. 2) Suppose that the action is semipositive and not positive at $x$ i.e. $T_{-}=\{0\}$ and $T_{0} \neq\{0\}$. Then for any $y \in T_{0}, X_{y}$ $=\left(y+T_{+}\right) \cap X$ is invariant under the action, where $y+T_{+}$is the translation of $T_{+}$by $y$. Further if it is not empty, then $\{y\} \times\{0\} \in X_{y}$ and in fact, as follows from (1), it coincides with the fixed point set on $X_{y}$. Hence if $\operatorname{dim}_{x} F=0$, then $X_{y}=\phi$ for all $y \neq 0$ sufficiently near to 0 so that we have $X \subseteq T_{+}$at $x$ and hence $T_{0}=\{0\}$, which is a contradiction. Thus $\operatorname{dim}_{x} F>0$. The other case can be treated in the same way.

Q.E.D.

Let $X$ be a compact complex manifold. Then the group Aut $X$ of biholomorphic automorphisms of $X$ has the natural structure of a complex Lie group acting biholomorphically on $X$. (cf. [12, III. 1.1]). Put $G$ $=$ Aut $X$ and let $(S 3$ be the Lie algebra of $G$, naturally identified with the space of holomorphic vector fields on $X$. Suppose now that $X$ is a Kähler manifold with the associated positive real $(1,1)$-form $\omega$ on $X$. Let $\mathbb{Q}$ $=\{V \in \mathbb{S} ; \operatorname{zero}(V) \neq \varnothing\}$. Then $\mathbb{R}$ forms a Lie subalgebra of $G$ (cf. [9, Prop. 6.8]). Let $L$ be the connected Lie subgroup of $G$ corresponding to $\mathcal{\Omega}, K^{\prime}$ the group of all isometries of $X$ (considering $X$ as a Riemannian manifold with metric $g$ corresponding to $\omega$ ), and $K$ the identity component of $K^{\prime}$. Then $K^{\prime}$ has the natural structure of a real compact Lie group, 
and its Lie algebra, $\boldsymbol{\Re}$, is identified with the set of Killing vector fields on $X$ with respect to $g$. Let $J$ be the tensor field of type $(1,1)$ on $X$ giving the complex structure of $X$. Then by a theorem of Yano [12, III. 4. 3] for any $V \in \mathfrak{\Omega}$ the complex vector field $\alpha(V)$ of type $(1,0)$ defined by $\alpha(V)=V-\sqrt{ }-1 J V$ is holomorphic. Hence $\alpha$ defines an injective map $\alpha: \Re \rightarrow(\mathfrak{S}$. Considering this as identification we may regard $\Re$ (resp. $K$ ) as a real Lie subalgebra (resp. subgroup) of $\$$ (resp. $G$ ). Let $\Omega_{0}$ $=\Re \cap \Omega$, which is the Lie algebra of $K_{0}=K \cap L$. Let $K_{1}$ be any compact Lie subgroup of $K_{0}$. Then its complexification $K_{1}^{C}$ can naturally be considered as a reductive Lie subgroup of $L$, and the fixed point sets of $K_{1}$ and $K_{1}^{C}$ coincide. In particular if $K_{1}$ is a real torus of real dimension $k$, then $K_{1}^{C}$ is an algebraic torus isomorphic to $\left(\mathbb{C}^{*}\right)^{k}$. Since every $V$ $\in \mathfrak{N}_{0}$ generates a real torus $T_{\mathbb{R}}$ such that zero $(V)=$ Fix $T_{\mathbb{R}}$, Fix $T_{\mathbb{R}}$ being the fixed point set of $T_{\mathbb{R}}$, we obtain the following:

Lemma 1. 4. Let $V$ be a Killing vector field on $X$ with nonempty zero set. Then there is a subgroup $T \subseteq$ Aut $X$, isomorphic to $\left(\mathbb{C}^{*}\right)^{k}$ for some $k$, such that $\operatorname{zero}(V)=\mathrm{Fix}(T)$.

Let $g$ be a Kähler metric on $X$ as above. Then for any real $C^{\infty}$ vector field $W$ on $X$ the real $C^{\infty} 1$-form associated with $W$ is the 1 -form $\zeta$ defined by the following condition; $\zeta_{x}\left(W_{x}^{\prime}\right)=g_{x}\left(W_{x}, W_{x}^{\prime}\right)$ for all $x \in X$ and for all $C^{\infty}$ vector fields $W^{\prime}$ on $X$. For later use we recall the following (cf. [12, III. 4.6]):

Lemma 1. 5. Let $V$ be as in Lemma 1.4, and $\xi$ the real 1-form associated with JV in the sense defined above. Then there is a real $C^{\infty}$ function $f$ on $X$ such that $\xi=d f$.

Remark 1.1. Suppose that $V$ generates a 1-dimensional torus $S^{1}$ and its complexification $\mathbb{C}^{*}$ acts on $X$. Identify $\mathbb{C}^{*}$ with $S^{1} \times \mathbb{R}^{+}$by the map $t \rightarrow(\arg t,|t|)$, where $\arg t$ is the argument of $t$. Then $J V$ is a vector field generating the action of $\mathbb{R}^{+}$. In a neighborhood of a fixed point as in (1) $J V$ is up to constants given by $\sum_{i} m_{i}\left(z_{i} \partial / \partial z_{i}+\bar{z}_{i} \partial / \partial \bar{z}_{i}\right)$, or in real coordinates $x_{i}=1 / 2\left(z_{i}+\bar{z}_{i}\right)$ and $v_{i}=1 / 2 \sqrt{-1}\left(z_{i}-\bar{z}_{i}\right), \quad J V$ 
$=\sum_{i} m_{i}\left(x_{i} \partial / \partial x_{i}+y_{i} \partial / \partial y_{i}\right)$. Note that with respect to the standard Euclidian metric $f$ would be given by $f=\sum_{i} m_{i}\left|z_{i}\right|^{2}$.

Let $\widetilde{V}=\alpha(V)=V-\sqrt{-1} J V$ be the holomorphic vector field associated with a Killing vector field $V$ on a compact Kähler manifold $X$ as above. Following Carrell and Lieberman [6] we consider the Koszul complex

$$
0 \rightarrow \Omega_{X}^{n} \rightarrow \cdots \rightarrow \Omega_{X}^{1} \rightarrow \mathcal{O}_{X} \rightarrow 0
$$

associated with $\widetilde{V}$, where the differential is given by the contraction $i_{\widetilde{V}}$ by $\widetilde{V}$. Set $K_{X}^{i}=\Omega_{\bar{X}}^{-i}$ in order to make the differential of degree +1 . Then the hypercohomology of the complex $K_{\dot{X}}$ gives rise to the following two spectral sequences

$$
\begin{aligned}
& E_{2}^{p, q}=H^{p}\left(X, H^{q}\left(K_{\dot{X}}\right)\right) \Rightarrow H^{p+q}\left(X, K_{\dot{X}}\right) \\
& E_{1}^{p, q}=H^{q}\left(X, \Omega_{\dot{X}}^{-p}\right) \Rightarrow \mathbb{H}^{p+q}\left(X, K_{\dot{X}}\right) .
\end{aligned}
$$

As was shown in the proof of Theorem 1 in [6] the second spectral sequence degenerates. Hence for every integer $m$ we have the inequality

$$
\sum_{q-p=m} \operatorname{dim} H^{q}\left(X, \Omega_{X}^{p}\right)=\operatorname{dim} \mathbb{H}^{m}\left(X, K_{X}\right) \leqq \sum_{p+q=m} \operatorname{dim} H^{p}\left(X, H^{q}\left(K_{\dot{X}}\right)\right) .
$$

Let $Z=$ zero $\widetilde{V}$ (=zero $V$ ) be the zero set of $\widetilde{V}$. We shall show that $H^{q}\left(K_{\dot{X}}\right)=\Omega_{\bar{Z}}^{-q}$ to obtain the following:

Lemma 1. 6. Suppose that $V$ generates $S^{1}$ as in Remark 1.1. Then for every integer $m$ we have the following inequality

$$
\sum_{q-p=m} \operatorname{dim} H^{q}\left(X, \Omega_{X}^{p}\right) \leqq \sum_{q-p=m} \operatorname{dim} H^{q}\left(Z, \Omega_{Z}^{p}\right)
$$

Corollary 1.7. For $a=0,1$ we have the following inequality

$$
\sum_{i \equiv a(2)} \operatorname{dim} H^{i}(X, \boldsymbol{C}) \leqq \sum_{i \equiv a(2)} \operatorname{dim} H^{i}(Z, \boldsymbol{C}) .
$$

Remark 1.2. The above corollary is a special case of the inequality of Conner (cf. [2, IV. 5. 4]) which was also used by Frankel in [8].

Proof of Lemma 1.6. As was mentioned above it suffices to show that $H^{q}\left(K_{\dot{X}}\right) \cong \Omega_{\bar{Z}}^{-q}$. Outside $Z$ this is wellknown. Hence we consider at points of $Z$. Since the problem is local, we may work in the local 
coordinates as in (1). So we assume that $X=\mathbb{C}^{n}\left(z_{1}, \cdots, z_{n}\right), Z$ is defined by the equation $z_{1}=\cdots=z_{r}=0$, and $\widetilde{V}=\sum_{i=1}^{r} m_{i} z_{i} \partial / \partial z_{i}$ for some integers $m_{i} \neq 0$ (cf. Remark 1.1 above). Let $Y=\left\{\left(z_{i}\right) ; z_{r+1}=\cdots=z_{n}=0\right\}$. Then we have the natural isomorphism $\Omega_{X, 0}=\dot{\oplus}_{s=0} \Omega_{z, 0}^{\dot{z}-s} \widehat{\otimes}_{C} \Omega_{Y, 0}^{s}$, and $i_{\widetilde{r}}=$ $\dot{\oplus}_{s=0} i d_{Z} \widehat{\otimes}_{\mathbb{C}} i \widetilde{v}$, where $\widehat{\otimes}$ is the analytic tensor product and on the right hand side $\widetilde{V}$ is also considered as a holomorphic vector field on $Y$ naturally. Hence we have only to show that the following complex

$$
0 \rightarrow \Omega_{Y, 0}^{r} \underset{i_{\widetilde{\vartheta}}}{\rightarrow} \cdots \underset{i_{\overparen{\vartheta}}}{\rightarrow} \mathcal{U}_{\Gamma, 0} \underset{\varepsilon}{\rightarrow} \mathbb{C} \rightarrow 0
$$

is exact, where $\varepsilon$ is the evaluation at 0 . In fact this is a special case of de Rham lemma cl. [14].

Q.E.D.

\section{§ 2. B.-B. Decomposition Associated with a $\mathbb{C}^{*}$ Action}

Fix once and for all the inclusion $\mathbb{C}^{*}(t) \subseteq \mathbb{C}(t) \subseteq \mathbb{P}^{1}=\mathbb{C}(t) \cup\{\infty\}$, where $\boldsymbol{P}^{1}$ is the complex projective line. Let $X$ be a connected complex space on which $\mathbb{C}^{*}$ acts biholomorphically. We call this action $\sigma$ : $\mathbb{C}^{*} \times X \rightarrow X$ meromorphic if $\sigma$ extends to a meromorphic map $\sigma^{*}: \mathbb{P}^{1} \times X \rightarrow X$ (cf. [9, Def. 2.1]). Suppose that $\sigma$ is meromorphic and $X$ is compact. Then for every $x \in X$ the orbit map $\sigma(x): \mathbb{C}^{*} \rightarrow X, \sigma(x)(g)=\sigma(g x)$, $g \in \boldsymbol{C}^{*}$, extends to a unique morphism $\sigma(x)^{*}: \mathbb{P}^{1} \rightarrow X$. Then we define the point $0(x)$ (resp. $\infty(x)) \in X$ by

$$
0(x)=\sigma(x) *(0) \quad(\text { resp. } \infty(x)=\sigma(x) *(\infty)) .
$$

For instance in Lemma 1.31 ) for every $z \in T_{+} \cap X$ (resp. $T_{-} \cap X$ ) we have that $0(z)=x$ (resp. $\infty(z)=x)$.

In order to state the following lemma we introduce the following terminology. By a decomposition of $X$ we mean a finite collection, $\left\{X_{\alpha}\right\}$, of mutually disjoint locally closed irreducible analytic subsets $X_{\alpha}$ of $X$, such that $Y=\cup_{\alpha} X_{\alpha}$. A decomposition $\left\{X_{a}\right\}$ is called $\mathbb{C}^{*}$ invariant if $\mathbb{C}^{*}$ leaves all the ${ }^{\alpha} X_{\alpha}$ invariant.

Lemma 2. 1. Let $X$ be a compact connected complex manifold on which $\mathbb{C}^{*}$ acts biholomorphically and meromorphically. Let $F$ be the set of fixed points of this $\mathbb{C}^{*}$ action, and $F_{\alpha}, 1 \leqq \alpha \leqq m$, the con- 
nected components of $F$. Then there exists a unique decomposition, $\left\{X_{\alpha}\right\}, 1 \leqq \alpha \leqq m$, of $X$ which is $\mathbb{C}^{*}$ invariant and with the following properties: For each $\alpha, X_{\alpha}$ contains $F_{\alpha}$ and there exists a $\mathbb{C}^{*}$-equivariant retraction $\pi_{a}: X_{\alpha} \rightarrow F_{\alpha}$, such that with respect to $\pi_{\alpha}, X_{\alpha}$ is a fiber boundle over $F_{\alpha}$ with typical fiber $\mathbb{C}^{*}$-equivariantly isomorphic to $\mathbb{C}^{\lambda_{\alpha}}$, where $\lambda_{\alpha}=\operatorname{dim} T_{+}(x)$ for all $x \in F_{\alpha}$ and $\mathbb{C}^{*}$ acts on $\mathbb{C}^{\lambda_{\alpha}}$ linearly and positively.

Proof. We define $X_{\alpha}$ by $X_{\alpha}=\left\{x \in X ; 0(x) \in F_{\alpha}\right\}$. Clearly $X$ is then written as a disjoint union $X=\cup_{\alpha} X_{\alpha}$ and $X_{\alpha}$ are $\mathbb{C}^{*}$ invariant. First we show that each $X_{\alpha}$ is a locally closed submanifold of $X$. Take an arbitrary point $x \in F_{\alpha}$. Set $n=\operatorname{dim} X, n_{\alpha}=\operatorname{dim} F_{\alpha}$ and $r_{\alpha}=n-n_{\alpha}$. Then by (1) in Section 1 , with respect to a suitable local coordinates $\left(z_{1}, \cdots, z_{n_{\alpha}}\right.$, $\left.w_{1}, \cdots, w_{r_{\alpha}}\right)$ defined in a neighborhood $V$ of $x$, the action $\sigma$ takes the form

$$
\sigma\left(t .\left(z_{1}, \cdots, z_{n_{\alpha}}, w_{1}, \cdots, w_{r_{\alpha}}\right)\right)=\left(z_{1}, \cdots, z_{n_{\alpha}}, t^{m_{1}} w_{1}, \cdots, t^{m_{r_{\alpha}} w_{r_{\alpha}}}\right),
$$

where $F_{\alpha}$ is defined by $w_{1}=\cdots=w_{r_{\alpha}}=0$ in $V$ and $m_{i} \neq 0$. Then if we arrange the coordinates in such a way that $m_{1} \geqq \cdots \geqq m_{\lambda_{\alpha}} \geqq 0 \geqq m_{\lambda_{\alpha+1}} \geqq \cdots \geqq$ $m_{r_{\alpha}}$, then it is clear that $X_{\alpha}$ is given in $V$ by the equation $w_{\lambda_{\alpha+1}}=\cdots=w_{r_{\alpha}}$ $=0$. It follows then that $X_{\alpha}$ is a submanifold of $X$ of dimension $\lambda_{\alpha}+n_{\alpha}$ in a neighborhood $W$ of $F_{\alpha}$. Since every point $x^{\prime} \in X$ is equivalent to a point of $W$ under the action of $\mathbb{C}^{*}$, this implies that $X_{\alpha}$ is a locally closed analytic submanifold of $X$.

Next we define $\pi_{a}$ by $\pi_{\alpha}(x)=0(x), x \in X_{\alpha}$. Then it is obvious that $\pi_{\alpha}$ is holomorphic and $\left.\pi_{\alpha}\right|_{F_{\alpha}}=i d_{F_{\alpha}}$. We show that $\pi_{\alpha}$ is a fiber bundle with fiber $\mathbb{C}^{\alpha_{\alpha}}$ as stated in the lemma. Let $x \in F_{\alpha}$ be as above. We have to show that for a small neighborhood $U$ of $x$ in $F_{\alpha}$ there is a $\mathbb{C}^{*}$ equivariant isomorphism $\pi_{\alpha}^{-1}(U) \cong U \times \mathbb{C}^{\lambda_{\alpha}}$, where $\mathbb{C}^{*}$ acts on $\mathbb{C}^{\lambda_{\alpha}}=$ $C^{\lambda_{\alpha}}\left(w_{1}, \cdots, w_{\lambda_{\alpha}}\right)$ by $\left(w_{1}, \cdots, w_{\lambda_{\alpha}}\right) \rightarrow\left(t^{m_{1}} w_{1}, \cdots, t^{m_{\lambda_{\alpha}}} w_{\lambda_{\alpha}}\right)$. In fact from the above local expression we have such an isomorphism locally, that is, if we replace $\mathbb{C}^{\lambda_{\alpha}}$ by $D^{\lambda_{\alpha}}=\left\{\left(w_{i}\right) \in \mathbb{C}^{\lambda_{\alpha}} ;\left|w_{i}\right| \leqq 1\right\}$ and $\pi_{\alpha}^{-1}(U)$ by a suitable neighborhood $V_{0}$ of $U$ in $\pi_{a}^{-1}(U)$. Then since every $x^{\prime} \in \pi_{\alpha}^{-1}(U)$ is equivalent by the $\mathbb{C}^{*}$ action to a point in $V_{0}$ as above, by an elementary argument we get easily the desired isomorphism. Finally by construction it is clear that $\lambda_{\alpha}=\operatorname{dim} T_{\mathrm{T}}(x)$ for all $x \in F_{a}$.

Q.E.D. 
The above construction is due to Bialynicki-Birula [1]. So we call the decomposition obtained in the above lemma the B.-B. (BialynickiBirula) decomposition of $\mathrm{X}$ with respect to the given $\mathbb{C}^{*}$ action. Note that using $\infty(x)$ instead of $O(x)$ we may obtain another decomposition which is 'dual' to the one obtained above (cf. [1]).

Let $X$ be a complex space. Then a subset $A$ of $X$ is called Zariski locally closed in $\mathrm{X}$ if it is a Zariski open subset of an analytic subset of $X$. We call a decomposition $\left\{X_{a}\right\}$ of $X^{-}$meromorphic if each $X_{a}$ is Zariski locally closed, or equivalently, the closure $X_{\alpha}^{*}$ of $X_{\alpha}$ is analytic in $X$ and $X_{a}$ is $Z$ ariski open in $X_{a}^{*}$. Then the rest of this section is devoted to proving the following:

Theorem 2. 2. Let X be a compact Kähler manifold on which $\mathbb{C}^{*}$ acts biholomorphically and meromorphically. Then the B.-B. decomposition, $\left\{X_{\alpha}\right\}$, of $X$ associated with this $\mathbb{C}^{*}$ action is meromorphic in the sense defined above.

That $X_{\alpha}^{*}$ is analytic will be shown in Proposition 2.8 using the Douady space of $X$. In order to show that $X_{\alpha}$ is Zariski open in $X_{\alpha}^{*}$ we need some preliminary considerations on the structure of the fibers of proper morphisms of relative dimension $I$ of complex spaces with $\mathbb{C}^{*}$ actions compatible with the morphisms (Lemmas 2.3-2.7), to which the problem is reduced by way of Proposition 2.8. Kähler condition becomes crucial in the proof of Proposition 2.10 and hence of Lemma 2.11. Proof of Theorem 2.2 will then be given at the end of this section. In the case of projective varieties the results are known and due to Bialynicki-Birula [1] (cf. Remarks 2.1 and 2.2 below).

Notation etc. In the following, for any morphism $f: Z \rightarrow T$ of complex spaces and for any analytic subset $A \subseteq T$ we denote by $f^{-1}(A)$ the inverse image with reduced structure; $f^{-1}(A)=\left(Z \times{ }_{T} A\right)_{\text {red }}$. Moreover for any point $t \in T$ we mean by a fiber over $t$ the reduced subspace $f^{-1}(t)$ of $Z$. On the other hand, the general fiber of $f$ is always considered with respect to the Zariski topology of $T$. For example 'the general fiber of $\int$ is irreducible' means that $f^{-1}(u)$ is irreducible for every $u \in U$ 
for some dense Zariski open subset $U$ of $T$.

Now let $f: Z \rightarrow T$ be a proper morphism of irreducible complex spaces. Suppose that each fiber of $f$ has (pure) dimension 1 and that there is a biholomorphic and meromorphic $\boldsymbol{C}^{*}$ action on $Z$ which is nontrivial and compatible with $f$ i.e. $f(g z)=f(z), g \in \mathbb{C}^{*}$ and $z \in Z$. We denote by $B$ the fixed point set of this action. Note that since $f$ is proper and the action is compatible with $f$, the points $0(z)$ and $\infty(z)$ can be defined analogously for every $z \in Z$.

Lemma 2. 3. Let $f: Z \rightarrow T$ and $B$ be as above. Then the following hold. 1) $B$ contains no irreducible components of the fibers of $f$. 2) There is a unique irreducible component, $B_{0}=B_{0}(f)$ (resp. $B_{\infty}=B_{\infty}$ $(f))$, of $B$ which is characterized by the following property; let $Z_{0}=\left\{z \in Z ; 0(z) \in B_{0}\right\}$ (resp. $Z_{\infty}=\left\{z \in Z ; \infty(z) \in B_{\infty}\right\}$ ). Then $Z_{0}$ (resp. $Z_{\infty}$ ) contains a nonempty Zariski open subset of $Z$. Moreover $B_{0}$ and $B_{\infty}$ are the only components of $B$ which are mapped surjectively onto $T$.

Proof. 1) Suppose the contrary and let $C$ be an irreducible curve contained in $B$ such that $f(C)$ is a point $t$ of $T$. Let $z \in C$ be a point not contained in any other component of $f^{-1}(t)$. Then as follows from Lemma 1.31 ) there is an orbit $\mathfrak{D}$ in $Z$ such that $z \notin \mathfrak{D}$ but its closure $\mathfrak{D}^{*}$ contains $z$. Since the action is compatible with $f$, $\mathfrak{D}^{*}$ must be contained in a fiber of $f$. Hence $f\left(\mathfrak{D}^{*}\right)=t$ and $\mathfrak{D}^{*}$ is an irreducible components of $f^{-1}(t)$. This contradicts our choice of $z$.

2) First assume that $Z$ is normal and the general fiber of $f$ is connected. Then there is a nonempty Zariski open subset $U$ of $T$ such that $\left.f\right|_{f^{-1}(U)}$ : $f^{-1}(U) \rightarrow U$ is smooth (by Bertini and the fact that the singular locus of $Z$ is of codimension 2 in $Z$ ). Over $U$ each fiber of $f$ is isomorphic to $\boldsymbol{P}^{1}$, as a compactification of $\mathbb{C}^{*}$. Hence $f^{-1}(u) \cap B=\{0\} \cup\{\infty\}$. We show that there is no irreducible component $B^{\prime}$ of $B$ such that $B^{\prime} \cap f^{-1}(u)$ $=\{0\} \cup\{\infty\}$ for all $u \in U$. In fact supposing that such a $B^{\prime}$ exists let $B_{\vdash}^{\prime}$ (resp. $\left.B_{-}^{\prime}\right)=\left\{b \in B^{\prime}\right.$; the action is semipositive (resp. seminegative) at $b$, Then it is clear that $B_{+}^{\prime} \cap f^{-1}(u)=\{0\}$ and $B_{-}^{\prime} \cap f^{-1}(u)=\{\infty\}$ for all $u \in U$. In particular $B_{+}^{\prime}(U)$ and $B_{-}^{\prime}(U)$ are open in $B$, where $B_{ \pm}^{\prime}(U)$ 
$=B_{ \pm}^{\prime} \cap f^{-1}(U)$. Thus the connected set $B^{\prime} \cap f^{-1}(U)$ is the disjoint union of two open subsets $B_{+}^{\prime}(U)$ and $B_{-}^{\prime}(U)$. This contradiction shows the above assertion. We shall denote by $\omega$ either 0 or $\infty$ in the following. Then from the above assertion we conclude easily that there is a unique irreducible component $B_{\omega}$ of $B$ such that for every $u \in U, B_{\omega} \cap f^{-1}(u)$ coincides with $\omega$ of $f^{-1}(u) \cong \boldsymbol{P}^{1}$. Clearly $B \cap f^{-1}(U)=\left(B_{0} \cup B_{\infty}\right) \cap$ $f^{-1}(U)$ Moreover we get that $Z_{0}\left(Z_{\infty}\right) \cap f^{-1}(U)=f^{-1}(U)-f^{-1}(U) \cap B_{\infty}$ $\left(B_{0}\right)$ and hence $Z_{\omega}$ contains a nonempty Zariski open subset of $Z$.

Next we consider the case where $Z$ is normal and $f$ is general.

Let $f=f_{2} f_{1}$, with $f_{1}: Z \rightarrow T_{1}$ and $f_{2}: T_{1} \rightarrow T$, be the Stein factorization of $f$, where every fiber of $f_{1}$ is connected and $f_{2}$ is a finite morphism. Then since $C^{*}$ is connected, the action is compatible also with $f_{1}$. Hence we can apply what we have proved above to $f_{1}$ to obtain $B_{\omega}$ with the desired properties. Finally in the general case let $n: \widetilde{Z} \rightarrow Z$ be the normalization of $Z$ and put $\tilde{f}=f n$. Then the given $\mathbb{C}^{*}$ action extends naturally to a biholomorphic and meromorphic $\mathbb{C}^{*}$ action on $\tilde{Z}$ which is compatible with $\tilde{f}$. Then apply to $\tilde{f}$ the above consideration to obtain $\widetilde{B}_{\omega}$ in $\widetilde{Z}$ with $\widetilde{Z}_{\omega}=\left\{\widetilde{z} \in \widetilde{Z} ; \omega(\widetilde{z}) \in \widetilde{B}_{\omega}\right\}$ containing a nonempty Zariski open subset of $\tilde{Z}$. Set $B_{\omega}=n\left(\widetilde{B}_{\omega}\right)$. Then $Z_{\omega}=n\left(\widetilde{Z}_{\omega}\right)$ and hence it also contains a nonempty Zariski open subset of $Z$ and $f\left(B_{\omega}\right)=n f\left(\widetilde{B}_{\omega}\right)=T$. Hence these $B_{\omega}, \omega=0, \infty$, are the desired ones.

Q.E.D.

For our later purpose it will be useful to consider the following additional condition (A) on $f: Z \rightarrow T$ as in Lemma 2.3:

(A) A1) Each connected component of the general fiber of $f$ is irreducible. A2) Each connected component of every fiber of $f$ is simply connected. A3) $B_{0}$ (resp. $B_{\infty}$ ) intersects with each connected component of all the fibers of $f$ at just one point, where $B_{0}$ (resp. $B_{\infty}$ ) is as in Lemma 2.3.

Then we want to study the structure of fibers of $f$ subject to the condition (A). To describe these we introduce some terminology. Let $C_{0}$ be a compact irreducible and simply connected rational curve. Suppose that $\mathbb{C}^{*}$ acts on $C_{0}$ biholomorphically and meromorphically and that the action is nontrivial. Then $C_{y}$, as a simply connerted compactification of $C^{*}$, has exactly two fixed points which may naturally be called 0 and 
$\infty ; 0=0(x)$ and $\infty=\infty(x)$ for any $x \neq 0, \infty$. Next let $C$ be a compact connected curve whose irreducible components are simply connected rational curves. Suppose that $C^{*}$ acts on $C$ biholomorphically and meromorphically and that the action is nontrivial on each irreducible component. Suppose further that there is a numbering, $C_{1}, \cdots, C_{m}$, of irreducible components of $C$ such that if $0_{i}$ and $\infty_{i}$ are the corresponding fixed points on $C_{i}$, then $C_{i} \cap C_{i+1}=\infty_{i}=0_{i+1}, i=1, \cdots, k-1$, and $C_{i} \cap C_{j}=\varnothing$ for $|i-j|$ $>1$. We shall call such a curve $C$ with $C^{*}$ action linear, and this numbering, which is obviously unique, the canonical numbering of the irreducible components of $C$.

Lemma 2.4. Let $f: Z \rightarrow T$ be as in Lemma 2.3. If each connected component of the general fiber of $f$ with the induced $\mathbb{C}^{*}$ action is linear, then each connected component of general fiber of $f$ is irreducible.

Proof. Taking Stein factorization we may assume that every fiber of $f$ is connected. Let $n: \widetilde{Z} \rightarrow Z$ be the normalization of $Z$ and $\tilde{f}=f n$. $\quad \tilde{Z}$ has the natural $\mathbb{C}^{*}$ action compatible with $\tilde{f}$. Let $V$ be a nonempty Zariski open subset of $T$ such that $\left.\tilde{f}\right|_{\tilde{f}^{-1}(V)}: \tilde{f}^{-1}(V) \rightarrow V$ is smooth and $f^{-1}(t)$ is linear for every $t \in V$. Let $\widetilde{B}_{\omega}=B_{\omega}(\tilde{f})$ for $\omega=0, \infty$. For any $t \in V$ let $\widetilde{C}_{1}(t), \cdots, \widetilde{C}_{q}(t)$ be the connected components of $\tilde{f}^{-1}(t)$ and $\widetilde{0}_{i}(t), \widetilde{\infty}_{i}(t)$ the corresponding fixed points on $\widetilde{C}_{i}(t)$, where $q$ is independent of $t \in V$. Then we have that $\widetilde{B}_{0} \cap \tilde{f}^{-1}(V)=\left\{\tilde{0}_{1}(t), \cdots, \tilde{0}_{q}(t)\right\}$ and $\widetilde{B}_{\infty} \cap \tilde{f}^{-1}(V)=\left\{\widetilde{\infty}_{1}(t), \cdots, \widetilde{\infty}_{q}(t)\right\}$. Let $C_{i}(t)=n\left(\widetilde{C}_{i}(t)\right)$. Then $0_{i}(t)=n\left(\tilde{0}_{i}(t)\right)$ and $\infty_{i}(t)=n\left(\widetilde{\infty}_{i}(t)\right)$ are the corresponding fixed points on $C_{i}(t)$. On the other hand, restricting $V$ if necessary, we may assume that $C_{i}(t) \neq C_{j}(t)$ for $i \neq j$. (More precisely take $V$ in such a way that either $\tilde{A} \cap \tilde{f}^{-1}(V)=\varnothing$ or $\left.\tilde{f}\right|_{\tilde{A}_{\cap \tilde{f}^{-1}\left(I^{\prime}\right)}}: \tilde{A} \cap \tilde{f}^{-1}(V) \rightarrow V$ is smooth, where $\widetilde{A}=n^{-1}(A)$ with $A$ the set of non-normal points of $Z$.) Then after renumbering if necessary we may assume that $\left\{C_{i}(t)\right\}$ is the canonical numbering of the irreducible components of the linear curve $C(t)=\cup_{i=1}^{q} C_{i}(t)$. (Note that $C(t)$ is connected.) Suppose that $q>1$. Then since $0_{2}(t)=\infty_{1}(t)$ and $B_{\omega}=n\left(\widetilde{B}_{\omega}\right), \omega=0, \infty$, we get that $B_{0} \cap B_{\infty} \cap f^{-1}(t)$ $\neq \varnothing$ for all $t \in V$ where $B_{\omega}=B_{\omega}(f)$. This implies that $B_{0}=B_{\infty}$. Thus 
$0_{1}(t) \in B_{\infty}$ and hence $0_{1}(t)=\infty_{j}(t)$ for some $j$, which contradicts the linearity of $f^{-1}(t)$. Thus $q=1$ and the general fiber is irreducible.

Q.E.D.

Lemma 2.5. Let $f: Z \rightarrow T$ and $B, B_{0}, B_{\infty}$ be as in Lemma 2. 3 . Suppose that $f$ satisfies the condition (A) defined above. Then the following are true. 1) The connected components of all the fibers of $f$ with the induced $\mathbb{C}^{*}$ actions are linear. 2) Let $C_{1}^{i}(t), \cdots, C_{q_{i t}}^{i}(t)$ be the canonically numbered irreducible components of a connected component $C^{i}(t)$ (which is linear by 1$)$ ) of $f^{-1}(t), t \in T$, and $0_{\mu}^{i}(t)$ and $\infty_{\mu}^{i}(t)$ the corresponding fixed points on $C_{\mu}^{i}(t)$. Then $B_{0} \cap C^{i}(t)=0_{1}^{i}(t)$ and $B_{\infty} \cap C^{i}(t)=\infty_{q_{i t}}^{i}(t)$. In particular $B_{0} \cap B_{\infty}=\varnothing$.

From 2) follows immediately the following:

Corollary 2.6. Let $f: Z \rightarrow T$ and $B_{0}, B_{\infty}$ be as in the above lemma. Denote by $A$ either $B_{0}$ or $B_{\infty}$. If there is at least one point $b \in A$ with $0(z)=b \quad($ resp. $\infty(z)=b)$ for some $z \neq b$, then $A=B_{0}$ (resp. $\left.B_{\infty}\right)$.

Proof of Lemma 2.5. Using Stein factorization we reduce the problem without difficulty to the case where every fiber is connected, and hence in particular the general fiber is irreducible by A1). So we assume this in the following. First we consider 1) and 2) in the special case where $T$ is a 1 -dimensional $\operatorname{disc} S=\{s ;|s|<\varepsilon\}$, and show that $f^{-1}(0)$ is linear and 2) is true for $t=0$. Let $C_{\mu}, 1 \leqq \mu \leqq q$, be the irreducible components of $f^{-1}(0)$. Then by the simply connectedness of $f^{-1}(0)$ and Lemma 2.31 ) each $C_{n}$ is a simply connected rational curve and we have on it the two canonical fixed points $0_{\mu}$ and $\infty_{\mu}$. We first note that for any fixed point $b \in B$ the $\mathbb{C}^{*}$ action at $b$ is neither positive nor negative. In fact, if it is positive (resp. negative), then as follows from (1) in Section 1 for all $z \in Z$ near $b$ we must have $b=0(z)$ (resp. $\infty(z)$ ). This is impossible because $f(b)=f(0(z))=f(\approx)$ so that $z \in f^{-1}(b)$. Next let $0_{\mu} \in C_{\mu}$ be such that $0_{\mu} \notin C_{\nu}$ for any $\nu \neq \mu$. We show that the given $\mathbb{C}^{*}$ action is semipositive at such a $0_{\mu}$. In fact if it is not the case, then by Lemma 
1.31 ) there is a curve $C$ with a point $z \in C, z \neq 0_{\mu}$ such that $\infty(z)$ $=0_{\mu}$. Since $f(C)=0$, we must have $C=C_{\mu}$ by our choice of $0_{\mu}$, while on $C_{\mu}$ clearly there is no point with the above property, which is a contradiction. Hence the action is semipositive at $0_{\mu}$. Since the action is nonpositive as we have already seen above, by Lemma 1.32 ) there is a 1-dimensional irreducible component $B^{\prime}$ of $B$ passing through $0_{\mu}$. Clearly $B^{\prime} \nsubseteq f^{-1}(0)$ and so $f\left(B^{\prime}\right)=S$. Hence by Lemma 2.32 ) either $B^{\prime}$ $=B_{0}$ or $B_{\infty}$, while the latter is easily seen to be impossible. Thus if such a point $0_{\mu}$ exists, by A3) $B_{0} \cap f^{-1}(0)=\left\{0_{\mu}\right\}$. Similarly we prove that if $\infty_{\mu^{\prime}} \in C_{\mu^{\prime}}$ is such that $\infty_{\mu^{\prime}} \notin C_{\nu}$ for any $\nu \neq \mu^{\prime}$, then $B_{\infty} \cap f^{-1}(0)$ $=\left\{\infty_{\mu^{\prime}}\right\}$. In particular we see that there is at most one $0_{\mu}$ (resp. $\infty_{\mu^{\prime}}$ ) with the above property.

From this, combined with the simply connectedness of $f^{-1}(0)$ and the fact that $C_{\mu} \cap C_{\nu} \subseteq\left\{0_{\mu}, \infty_{\mu}\right\}$, it follows by an elementary consideration that there is exactly one such $0_{\mu}$ (resp. $\infty_{\mu^{\prime}}$ ), and indeed, more precisely that there is a unique numbering, still denoted by $\left\{C_{\mu}\right\}$, of the irreducible components of $f^{-1}(0)$ such that $0_{1}$ (resp. $\infty_{q}$ ) is such a fixed point, $C_{\mu} \cap C_{\mu+1}$ consists of one point, say $b_{\mu}, 1 \leqq \mu<q$, and $C_{\mu} \cap C_{\nu}=\varnothing$ for $|\mu-\nu|>1$. We then show that the $C^{*}$ action is not semipositive (resp. seminegative) at any $b_{\mu}$. In fact, otherwise, using Lemma 1.3 , we get as above that $B_{0} \cap f^{-1}(0)=\left\{b_{\mu}\right\}$ or $B_{\infty} \cap f^{-1}(0)=\left\{b_{\mu}\right\}$, which implies that $b_{\mu}=0_{1}$ or $\infty_{q}$ by A3), a contradiction. Hence by Lemma 1.31 ) there is a curve $D_{\mu}$ (resp. $D_{\mu}^{\prime}$ ) with a point $z \in D_{\mu}$ (resp. $z^{\prime} \in D_{\mu}^{\prime}$ ) $z \neq b_{\mu}$ (resp. $z^{\prime} \neq b_{\mu}$ ) such that $0(z)$ (resp. $\left.\infty(z)\right)=b_{\mu}$. It is clear that $\left\{C_{\mu}, C_{\mu+1}\right\}$ $=\left\{D_{\mu}, D_{\mu}^{\prime}\right\}$ as sets. Then by induction on $\mu$ we infer readily that $C_{\mu}$ $=D_{\mu}^{\prime}$ and $C_{\mu+1}=D_{\mu}$. This shows our final point; $b_{\mu}=\infty_{\mu}=0_{\mu+1}$. Hence $f^{-1}(0)$ is linear and 2) is established for $t=0$.

Using this result we now prove 1 ) and 2) in the general case. Let $t \in T$ be an arbitrary point and take a morphism $h: S \rightarrow T$ of a disc $S$ into $T$ such that $h(0)=t$, and that the induced morphism $f_{S}: Z_{S \text {, red }} \rightarrow S$ has irreducible fibers over $S^{\prime}=S-\{0\}$, where $Z_{S}=Z \times{ }_{T} S$ and red denotes taking the underlying reduced subspace. Suppose first that $T$ is locally irreducible at $t$. Then $Z_{S, r e d}$ is irreducible and clearly we get that $B_{0}$ $\times_{T} S=B_{0}\left(f_{S}\right) \quad$ (resp. $\left.B_{\infty} \times{ }_{T} S=B_{\infty}\left(f_{S}\right)\right)$. Thus we can apply the above consideration to $f_{S}$ to obtain 1) and 2) for $f$. Next in the general case 
let $T_{i}=T_{i}(t), i=1, \cdots, d$, be the local irreducible components of $T_{i}$ at $t$ defined in a neighborhood $W$ of $t$ in $T$. Corresponding to these we have the irreducible components $Z_{i}=Z_{i}(t)$ of $f^{-1}(W)$ characterized by the property that $f\left(Z_{i}\right)=T_{i}$. Let $f_{i}=\left.f\right|_{Z_{i}}: Z_{i} \rightarrow T_{i}$. Then one sees immediately that all the $f_{i}$ satisfy the condition (A). Hence by what we have proved above $f_{i}^{-1}(t)$ are linear and 2$)$ is true for $f_{i}^{-1}(t)$ for all $i$. Let $C_{1}^{i}, \cdots, C_{q_{i}}^{i}$ be the canonically numbered irreducible components of $f_{i}^{-1}(t)$ and $0_{j}^{i}$ and $\infty_{j}^{i}$ the corresponding fixed points on $C_{j}^{i}$. Then by 2 ) for $f_{i}$ and A3) for $f$, we have that $0_{1}^{1}=0_{1}^{2}=\cdots=0_{1}^{d}$ and $\infty_{q_{1}}^{1}=\cdots=\infty_{q_{d}}^{d}$. (Note that $B_{\omega} \cap f^{-1}(W)=\cup_{i} B_{\omega}\left(f_{i}\right), \omega=0, \infty$.) Then from the simply connectedness of $f^{-1}(t)$, we get that $f^{-1}(t)=f_{1}^{-1}(t)=\cdots=f_{d}^{-1}(t)$ as subsets of $f^{-1}(t)$. Hence the above $Z_{s, \text { red }}$ is also irreducible in the general case and we then obtain 1) and 2) for $f$ as above.

Q.E.D.

Let $f: Z \rightarrow T$ and $B$ be as in Lemma 2.3. Let $\left\{B_{i}\right\}$ be the set of irreducible components of $B$. We then consider the following condition $(\tilde{\mathrm{A}})$ on $f$, more restrictive than (A) defined above:

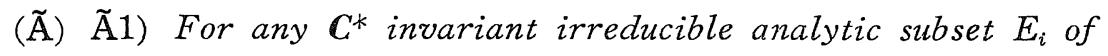
$Z$ such that $B_{i \omega}=B_{i}$, the induced morphism $f_{i}=\left.f\right|_{E_{i}}: E_{i} \rightarrow T_{i}$ with the induced $\mathbb{C}^{*}$ action satisfies the condition (A), where $B_{i \omega}=B_{\omega}\left(f_{i}\right), \omega$ $=0$ or $\infty$, and $T_{i}=f\left(E_{i}\right)$. In particular $f$ satisfies the condition $(\mathrm{A})$. Ã2) For $\omega=0, \infty, B_{i} \cap B_{\omega}=\varnothing$ if $B_{i} \neq B_{\omega}$.

Lemma 2. 7. Let $f: Z \rightarrow T$ and $B$ be as in Lemma 2.3. Suppose that $Z$ and $T$ are compact, and that $f$ satisfies the condition ( $\tilde{\mathrm{A}}$ ) above. Then the set $Z_{0}$ defined in Lemma 2.3 is Zariski open in $Z$.

Proof. Let $\left\{B_{0}, B_{1}, \cdots, B_{d}, B_{\infty}\right\}$ be the set of the irreducible components of $B$, where $B_{0}=B_{0}(f)$ and $B_{\infty}=B_{\infty}(f)$. For each $B_{i}, i=1, \cdots$, $d, \infty$, let $T_{i}=f\left(B_{i}\right)$ and $Z_{i}=f^{-1}\left(T_{i}\right)$. We show that there are $\mathbb{C}^{*}$ invariant irreducible analytic subsets $E_{i}$ of $Z_{i}, i=1, \cdots, d, \infty$, such that 1 ) $\left.B_{i}=B_{0}\left(f_{E_{i}}\right), i \neq \infty, 2\right)$ if $0(z) \in B_{i}$ for some $z \in Z$, then $z \in E_{i}$ and 3) $E_{i} \cap B_{0}=\varnothing$, where $f_{E_{i}}=f i_{E_{i}}: E_{i} \rightarrow T_{i}$. We first show that 2), 3) imply that $Z_{0}=Z-\cup_{i} E_{i}$. In fact by 3$)$ and the definition of $Z_{0}$ it follows immediately that $Z_{0} \subseteq Z-\bigcup_{i} E_{i}$. Conversely if $z \notin \bigcup_{i} E_{i}$, then by 2) $0(z)$ 
$\in B_{0}$ so that $z \in Z_{0}$. This shows the above equality. In particular $Z_{0}$ is Zariski open in $Z$.

Thus for the lemma it suffices to show the existence of $E_{i}$ as above. We put $E_{\infty}=B_{\infty}$. Then noting that $f$ satisfies the condition of Lemma 2. 5, by Corollary 2.6 and Lemma 2.52 ) we see that this satisfies the conditions 2) and 3) above respectively. So assume that $i \neq \infty$. For every $t \in T$ let $C_{1}(t), \cdots, C_{q_{t}}(t)$ and $0_{\mu}(t), \infty_{\mu}(t)$ be as in Lemma 2.52 ) (omitting the suffix $i$ since the fibers are connected). Let $Z_{i \lambda}$ be the irreducible components of $Z_{i}, f_{i \lambda}=\left.f\right|_{z_{i \lambda}}: Z_{i \lambda} \rightarrow T_{i}$ and $B_{i \lambda \omega}=B_{\omega}\left(f_{i \lambda}\right), \omega=0, \infty$. Suppose that $B_{i} \subseteq Z_{i \lambda}$ for some $\lambda$. Then since $f\left(B_{i}\right)=T_{i}$, by Lemma 2.3 2) $B_{i}=B_{i \lambda 0}$ or $B_{i \lambda \infty}$. Let $b \in B_{i}$ be an arbitrary point. Then $b=0_{\mu}(t)$ $=\infty_{\mu-1}(t)$ for a unique $1<\mu=\mu(b) \leqq q_{t}, t=f(b)$, for by Lemma 2.52 ) and $\tilde{\mathrm{A}} 2) B_{0} \cap f^{-1}(t)=0_{1}(t), B_{\infty} \cap f^{-1}(t)=\infty_{q_{t}}$ and $B_{0} \cap B_{i}=B_{\infty} \cap B_{i}=\varnothing$. So $Z_{i \lambda}$ contains at least one of $C_{\mu-1}(t)$ and $C_{\mu}(t)$. If $Z_{i \lambda} \supseteqq C_{\mu}(f(b))$ for some $b \in B$, then by Corollary 2.6 applied to the morphism $f_{i x}$ we get that $B_{i}=B_{i \lambda 0}$. Then again by Corollary 2.6 this implies that $Z_{i x}$ $\cap C_{\mu-1}(f(b))=\infty_{\mu-1}(f(b))$ so that $Z_{i \lambda} \not C_{\mu-1}(f(b))$ and hence $Z_{i \lambda}$ $\supseteqq C_{\mu}(f(b))$ for all $b \in B_{i}$, where $\mu=\mu(b)$. From this we infer readily that there is a unique irreducible component $Z_{i \lambda}$ containing $B_{i}$ such that $B_{i}=B_{i \lambda 0}$. We put $E_{i}=Z_{i \lambda}$ for this $\lambda$.

We shall check the conditions 2) and 3) above. 2) If $0(z)=b \in B_{i}$, then $z \in C_{\mu}(f(b)) \subseteq E_{i}$ by the definition of $Z_{i i}$. 3) Suppose that $\exists b$ $\in B_{0} \cap E_{i}$. Then $b=0_{1}(t), t=f(b)$, by Lemma 2.52$)$. Let $C^{0}(t)$ be the connected component of $f_{E_{i}}^{-1}(t)$ containing $b$. Let $C_{i}^{0}, i=1, \cdots, \nu$, be the canonically numbered irreducible components of $C^{0}(t)$. Then since $0_{1}(t)$ $\in C^{0}(t)$, it is clear that $C_{1}^{0}(t)=C_{1}(t)$. Hence applying Lemma 2.52 ) to $f_{E_{i}}$, we get that $0_{1}(t) \in B_{0}\left(f_{E_{i}}\right)=B_{i}$. Hence $B_{i} \cap B_{0} \neq \varnothing$, which contradicts $\tilde{\mathrm{A}} 2)$. Thus $B_{0} \cap E_{i}=\varnothing$.

Q.E.D.

Let $X$ be a compact complex space. Then we write $X \in \mathscr{C}$ if there is a compact Kähler manifold $Y$ and a surjective meromorphic map $g$ : $Y \rightarrow X$ (cf. [9, Def. 1. 1]).

Proposition 2. B. Let $X$ be a compact complex manifold with $X \in \mathscr{C}$. Suppose that $\mathbb{C}^{*}$ acts biholomorphically and meromorphically 
on $X$. Let $\left\{X_{a}\right\}$ be the B.-B. decomposition of $X$ with respeci to this $\mathbb{C}^{*}$ action. Then for each $\alpha$ there is a diagram

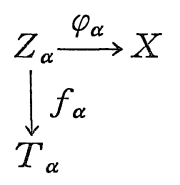

with the following properties: 1) $f_{\alpha}$ is a flat morphism of irreducible compact complex spaces $Z_{\alpha}$ and $\left.T_{\alpha}, 2\right) \varphi_{\alpha}$ is a bimeromorphic morphism of $Z_{\alpha}$ onto the closure $X_{\alpha}^{*}$ of $X_{\alpha}$ (in particular $X_{\alpha}^{*}$ is analytic by a theorem of Remmert) such that the restriction, $\left.\varphi_{\alpha}\right|_{Z_{\alpha t}}: Z_{\alpha t} \rightarrow X$, of $\varphi_{\alpha}$ to each fiber $Z_{a t}=f_{\alpha}^{-1}(t), t \in T_{\alpha}$, is an embedding, 3) there is a natural biholomorphic and meromorphic action of $\mathbb{C}^{*}$ on $Z_{\alpha}$, compatible with $f_{\alpha}$ and making $\varphi_{\alpha} \mathbb{C}^{*}$-equivariant, and finally 4) there is a dense Zariski open subset $U$ of $T_{\alpha}$ such that for every $t \in U, \varphi_{\alpha}\left(Z_{\alpha t}\right)$ is a closure of a regular, i.e. 1-dimensional, orbit in $X$.

Proof. The main line of the proof is almost analogous to [9, Lemma 4. 2] and we give only the necessary modifications in detail. We consider the induced $\mathbb{C}^{*}$ action on the Douady space $D_{X}$ of compact subspaces of $X$, and then its fixed point set $T$ on $D_{X}$. We then consider those irreducible components $T_{\nu}$ of $T$ such that for some $t \in T_{\nu}$ the corresponding subspace $Z_{t}$ of $X$ is reduced and is a closure of regular orbit contained in $X_{\alpha}$. Then using [9, Theorems 1.2 and 1.4] as in the proof of [9, Lemma 4.2] we can show that there is some index (call it $\alpha$ ) such that the restriction, $f_{\alpha}: Z_{\alpha} \rightarrow T_{\alpha}$, of the universal family $Z_{X} \rightarrow D_{X}$ to $T_{\alpha}$ has the properties 1), 3), 4) and also 2) with " $\varphi_{\alpha}=\left.\varphi\right|_{z_{\alpha}}$ being a bimeromorphic morphism onto $X_{\alpha}^{*}$ " replaced by " $\varphi_{\alpha}\left(Z_{\alpha}\right)$ containing an open subset of $X_{\alpha}$ ", where $\varphi: Z_{X} \rightarrow X$ is the natural projection.

Thus it remains to show that $\varphi_{\alpha}\left(Z_{\alpha}\right)=X_{\alpha}^{*}$ and $\varphi_{\alpha}$ is bimeromorphic onto its image. Note first that by virtue of 1 ), 2) and 4 ) the above $f_{\alpha}$ satisfies the conditions of Lemma 2.3. Let $B_{0}=B_{0}\left(f_{\alpha}\right)$. Then since $\left.\varphi_{\alpha}\right|_{z_{\alpha}}, t \in T_{\alpha}$, is a $\mathbb{C}^{*}$ equivariant embedding, we must have that $\varphi_{\alpha}\left(B_{0}\right.$ $\left.\cap Z_{\alpha t_{\alpha}}\right) \subseteq F_{\alpha}$, provided that $t_{\alpha}$ is a point of $T_{\alpha}$ such that $\varphi_{\alpha}\left(Z_{\alpha t_{\alpha}}\right)$ is a closure of a regular orbit contained in $X_{\alpha}$. Since $\varphi_{\alpha}\left(B_{0}\right) \subseteq F$ and it is connected, we must then have that $\varphi_{\alpha}\left(B_{0}\right) \subseteq F_{\alpha}$. Now let $Z_{0}$ be defined 
as in Lemma 2.3. Then since $\varphi_{\alpha}$ is $\mathbb{C}^{*}$ equivariant, by the definition of $X_{\alpha}$ we get that $\varphi_{\alpha}\left(Z_{0}\right) \subseteq X_{\alpha}$. This in turn implies that $\varphi_{\alpha}\left(Z_{\alpha}\right) \subseteq X_{\alpha}^{*}$ since $Z_{0}$ contains a nonempty Zariski open subset of $Z_{\alpha}$. On the other hand, the analytic set $\varphi_{\alpha}\left(Z_{\alpha}\right)$ contains an open subset of $X_{\alpha}$ and $X_{\alpha}$ is a connected locally closed submanifold of $X$, from which we deduce easily that $\varphi_{\alpha}\left(Z_{\alpha}\right)$ contains the whole $X_{\alpha}$. Hence $\varphi_{\alpha}\left(Z_{\alpha}\right)=X_{\alpha}^{*}$. That $\varphi_{\alpha}$ is bimeromorphic then follows quite as in the proof of [9, Lemma 4.2] and we omit it.

Q.E.D.

Our next aim is to show that $f_{\alpha}$ in the above proposition satisfy the condition ( $\widetilde{\mathrm{A}}$ ) so that Lemma 2.7 is applicable to $f_{\alpha}$. First we introduce some terminology. Let $f: Z \rightarrow T$ and $B$ be as in Lemma 2.3. Then a sequence of points $\left(z_{1}, \cdots, z_{q}\right)$ of $Z$ is said to generate a quasi-linear curve if $z_{i} \notin B$ for any $i, 0\left(z_{i}\right) \neq 0\left(z_{j}\right)$ for $i \neq j$ and $\infty\left(z_{i}\right)=0\left(z_{i+1}\right)$ for all $1 \leqq i \leqq q-1$. (We allow the case $q=1$ ). In fact in this case if $C_{i}$ is the closure of the orbit of $z_{i}, C=\bigcup_{i=1}^{q} C_{i}$ is a linear curve on $Z$ if $0\left(z_{1}\right) \neq \infty\left(z_{q}\right)$. Conversely, given any linear curve $C=\bigcup_{i=1}^{q} C_{i}$ we can obviously find a sequence $\left(z_{1}, \cdots, z_{q}\right)$ generating the linear curve $C$ with $z_{i} \in C_{i}$. Now we call $\left(z_{i}\right)$ as above generating a cycle (resp. a linear curve) if further $0\left(z_{1}\right)=\infty\left(z_{q}\right)$ (resp. $0\left(z_{1}\right) \neq \infty\left(z_{q}\right)$ ). Note that the above definitions also make sense on any compact complex space $X$ on which $\mathbb{C}^{*}$ acts biholomorphically and meromorphically.

Lemma 2.9. Let $f: Z \rightarrow T$ and $B$ be as in Lemma 2.3. Suppose that $f$ is flat and the general fiber of $f$ is irreducible. Let $t \in T$ be any point. 1) If a connected component of $f^{-1}(t)$ is not simply connected, there is a sequence of points $\left(z_{1}, \cdots, z_{q}\right)$ of $f^{-1}(t)$ which generates a cycle. 2) Suppose that $f^{-1}(t)$ is simply connected. Then for any couple of points $b_{1}, b_{2} \in B$ contained in one and the same connected components of $f^{-1}(t)$, there is a sequence $\left(z_{1}, \cdots, z_{q}\right)$ of points of $f^{-1}(t)$ generating a linear curve such that $\left\{b_{1}, b_{2}\right\}=\left\{0\left(z_{1}\right), \infty\left(z_{q}\right)\right\}$.

Proof. Taking a base change as in the proof of Lemma 2.5 and using the flatness of $f$ we reduce the problem to the case where $T$ is a 1-dimensional disc $S$ and $t$ is the origin 0 of $S$. Let $n: \widetilde{Z} \rightarrow Z$ be the 
normalization of $Z$ and $\tilde{f}=f n: \widetilde{Z} \rightarrow S$ the induced morphism. There is a natural lifting of the $C^{*}$ action to $\tilde{Z}$ compatible with $\tilde{f}$. Since the singular locus of $\widetilde{Z}$ is isolated, restricting $S$ if necessary, we may assume that $\tilde{f}$ is smooth over $S^{\prime}=S-\{0\}$, and hence that $\tilde{f}^{-1}(s) \cong \mathbb{P}^{1}$ for all $s \in S^{\prime}$. We show that $\tilde{f}^{-1}(0)$ is simply connected. In fact, let $\lambda: Z^{\prime} \rightarrow \widetilde{Z}$ be a resolution of $\tilde{Z}$. Then as is well-known in the theory of analytic surfaces $Z^{\prime}$ is obtained from $S \times \mathbb{P}^{1}$ by a finite number of $\sigma$-processes (=blowing up points). Hence $(\lambda \tilde{f})^{-1}(0)$ is simply connected. Since by the normality of $\widetilde{Z}, \lambda$ is a contraction to the points of a union of some of the irreducible components of $(\lambda \tilde{f})^{-1}(0), \tilde{f}^{-1}(0)$ also is simply connected. Thus $\tilde{f}^{-1}(s)$ are simply connected for all $s \in S$. Then by Lemma 2.5 1) $\widetilde{C}=\tilde{f}^{-1}(0)$ is linear. (It is clear that $\tilde{f}$ satisfies A1) and A3).) Let $\widetilde{C}_{1}, \cdots, \widetilde{C}_{k}$ be the canonical numbering of irreducible components of $\widetilde{C}$ and $\widetilde{O}_{i}, \widetilde{\infty}_{i}$ the corresponding fixed points on $\widetilde{C}_{i}$.

Now we show 1$)$. Since $\widetilde{C}$ is linear, it is easily seen that $f^{-1}(0)$ is simply connected if and only if $\left.n\right|_{\widetilde{\sigma}}$ is a bijection of $\widetilde{C}$ and $C=f^{-1}(0)$. So take a point $b \in C$ such that $n^{-1}(b)$ contains more than one point. We may assume that $b \in B$. Then $n^{-1}(b)$ is a subset of $\left\{\widetilde{0}_{1}, \cdots, \tilde{0}_{q}, \tilde{0}_{q+1}\right\}$ where $\tilde{0}_{q\llcorner 1}=\widetilde{\infty}_{q}$. Take $\tilde{0}_{i_{1}}, \tilde{0}_{i_{2}} \in n^{-1}(b), i_{1}<i_{2}$ in such a way that $\tilde{0}_{i} \notin n^{-1}(b)$ for any $i_{1}<i<i_{\varepsilon}$. Take points $\widetilde{z}_{i} \in \widetilde{C}_{i}-\left\{\tilde{0}_{i}, \widetilde{\infty}_{i}\right\}$ one for each $i_{1} \leqq i<i_{2}$ and put $n\left(\widetilde{z}_{i}\right)=z_{i}$. Then it is obvious that the sequence $\left(z_{i_{1}}, \cdots, z_{i_{2}-1}\right)$ generates a cycle. 2) In the above notation take any $\tilde{0}_{i_{u}} \in n^{-1}\left(b_{u}\right), u=1,2$. We may assume that $i_{1}<i_{2}$. Then the sequence $\left(z_{i_{1}}, \cdots, z_{i_{2}-1}\right)$ defined as above generates a linear curve with $0\left(z_{i_{1}}\right)=b_{1}$ and $\infty\left(z_{i_{2}-1}\right)=b_{2}$ since $f^{-1}(t)$ is simply connected.

Q.E.D.

Proposition 2.10. Let $X$ be a compact Kähler manifold on which $C^{*}$ acts biholomorphically and meromorphically. Let $F$ be the set of fixed points of this action. Then there is no finite sequence $\left(x_{1}, \cdots, x_{q}\right)$ of points of $X$ generating a quasi-linear curve with $0\left(x_{1}\right)$ and $\infty\left(x_{q}\right)$ belonging to one and the same connected component $F_{\alpha}$ of $F$. In particular there is no sequence $\left(x_{i}\right)$ generating a cycle on $X$.

Proof. Identify $C^{*}$ naturally with $S^{1} \times \mathbb{R}^{+}$as in Remark 1. 1. Let $V^{\prime}$ be a real vector field which is uniquely determined up to constants 
and generates the action of $\mathbb{R}^{+}$, and $\xi$ the real 1 -form associated with $V^{\prime}$ with respect to the given Kähler metric. Then by Lemma 1.5 and Remark 1.1 there is a real valued $C^{\infty}$ function $f$ such that $\xi=d f$. This implies that for every $x \in X-F$ the level set $f^{-1} f(x)$ is orthogonal at $x$ to the orbit $\mathbb{R}^{+} x$ with respect to the given Kähler metric. Hence if a variable point $y$ on $X$ moves from $0(x)$ to $\infty(x)$ along the orbit $\mathbb{R}^{+} x$, then $f$ either strictly increases or decreases from $f(0(x))$ to $f(\infty(x))$. (In fact one checks easily that $f$ strictly increases. cf. Remark 1.1.) Now suppose that there is a sequence $x_{1}, \cdots, x_{q}$ as stated in the lemma. Then we must have that $f\left(0\left(x_{1}\right)\right)<\cdots<f\left(0\left(x_{q}\right)\right)<f\left(\infty\left(x_{q}\right)\right)$ (resp. $f\left(0\left(x_{1}\right)\right)<f\left(\infty\left(x_{1}\right)\right)$ if $\left.q=1\right)$. But this is a contradiction since both $0\left(x_{1}\right)$ and $\infty\left(x_{q}\right)$ belong to $F_{\alpha}$ and $f$ is constant on $F_{\alpha}$ by the definition. (Note that $V^{\prime}$ vanishes on $F_{\alpha}$ )

Q.E.D.

Lemma 2.11. Let $X$ be as in the above proposition. Let $\left\{X_{\alpha}\right\}$ be the B.-B. decomposition associated with the given $\mathbb{C}^{*}$ action and $f_{\alpha}: Z_{\alpha} \rightarrow T_{\alpha}$ as in Proposition 2.8. Then for each $\alpha$, $f_{\alpha}$ satisfies the condition ( $\tilde{A})$ (defined just before Lemma 2.7).

Proof. First we check the condition (A) for $f_{\alpha}$ itself. A1) By 4) of Proposition 2.8 the general fibers of $f_{\alpha}$ are irreducible. A2) Suppose that $f_{\alpha}^{-1}(t)$ is not simply connected for some $t \in T_{\alpha}$. Then by Lemma 2.9 there is a sequence $\left(z_{1}, \cdots, z_{q}\right)$ of points of $f_{\alpha}^{-1}(t)$ which generates a cycle. Let $x_{k}=\varphi_{\alpha}\left(z_{k}\right)$. Then $\varphi_{\alpha}$ being $\mathbb{C}^{*}$ equivariant, the sequence $\left(x_{1}, \cdots, x_{q}\right)$ generates a cycle on $X$, which is impossible by Proposition 2. 10. A3) Suppose that $B_{0} \cap f_{\alpha}^{-1}(t)=\left\{b_{1}, b_{2}, \cdots\right\}, b_{1} \neq b_{2}$, for some $t \in T_{\alpha}$. Then by Lemma 2.9 there is a sequence $\left(z_{1}, \cdots, z_{q}\right)$ of points of $f_{\alpha}^{-1}(t)$ which generates a linear curve and with, say, $0\left(z_{1}\right)=b_{1}$ and $\infty\left(z_{q}\right)=b_{2}$. Then as above the sequence $\left(x_{1}, \cdots, x_{q}\right)$ with $x_{i}=\varphi_{\alpha}\left(z_{i}\right)$ generates a quasilinear curve on $X$ with $0\left(x_{1}\right), \infty\left(x_{q}\right) \in \varphi_{\alpha}\left(B_{0}\right)=F_{\alpha}$, which is impossible by Proposition 2.10. Similarly using the fact that $\varphi_{\alpha}\left(B_{\infty}\right)$ is contained in one and the same connected component of $F$, we prove that $B_{\infty} \cap f_{\alpha}^{-1}(t)$ consists of a single point for all $t \in T_{\alpha}$. Hence $f_{\alpha}$ satisfies the condition (A). In particular every fiber of $f_{\alpha}$ is linear by Lemma 2. 5 . 
Now let $\left\{B_{i}\right\}$ be the set of irreducible components of $B$. Let $E_{i}$ be any $\mathbb{C}^{*}$ invariant irreducible analytic subset of $Z$ with $B_{i \omega}=B_{i}, \omega=0$ or $\infty$, for some $i$, where $B_{i \omega}=B_{\omega}\left(f_{i}\right)$ with $f_{i}=\left.f\right|_{E_{i}}: E_{i} \rightarrow T_{i}=f\left(E_{i}\right)$. Then we show that $f_{i}$ also satisfies the condition (A). A2) Since every fiber $f_{i}^{-1}(t), t \in T_{i}$, is a union of irreducible components of $f_{\alpha}^{-1}(t)$, it follows that the connected components of $f_{i}^{-1}(t)$ are also linear and hence in particular simply connected. A1) then follows from Lemma 2.4. Finally using the linearity of the connected components of $f_{i}$ and Proposition 2.10 the proof of A3) is obtained in the same way as the proof of A3) for $f_{\alpha}$ above, since $\varphi_{\alpha}\left(B_{i}\right)$ is contained in one and the same $F_{\beta}$ for some $\beta$. Thus $f_{\alpha}$ satisfies $\tilde{\mathrm{A}} 1$ ).

We shall show $\tilde{\mathrm{A}} 2)$. Suppose that $B_{j} \cap B_{\omega} \neq \varnothing, B_{j} \neq B_{\omega}$, for some $j$ and $\omega=0$ or $\infty$. We assume that $\omega=0$, since the other case can be treated similarly. Fix a point $t \in f_{\alpha}\left(B_{j}\right)-f_{\alpha}\left(B_{j} \cap B_{0}\right)$. Let $C_{i}(t)$, $i=1, \cdots, q_{t}$, be the canonically numbered irreducible components of $f^{-1}(t)$ and $0_{i}(t)$ and $\infty_{i}(t)$ the corresponding fixed points on $C_{i}(t)$. Then by Lemma 2.52$) B_{0} \cap f^{-1}(t)=0_{1}(t)$. Let $b \in B_{j} \cap f^{-1}(t)$ be any point. Then $b=0_{k}(t)$ for some $1<k \leqq q_{t}+1$, where $0_{q_{t}+1}=\infty_{q_{t}}$. Take any points $z_{i} \in C_{i}-\left\{0_{i}\right\} \cup\left\{\infty_{i}\right\}$ for each $1 \leqq i<k$ and put $x_{i}=\varphi_{\alpha}\left(z_{i}\right)$. Then it is clear that the sequence $\left(x_{1}, \cdots, x_{k-1}\right)$ generates a linear curve on $X$ and $x_{1} \in \varphi_{\alpha}\left(B_{0}\right)$ and $x_{k-1} \in \varphi_{\alpha}\left(B_{j}\right)$. On the other hand, since $B_{0}$ $\cup B_{j}$ is connected, $\varphi_{\alpha}\left(B_{0}\right)$ and $\varphi_{\alpha}\left(B_{j}\right)$ must be contained in the same connected component $\left(=F_{\alpha}\right)$ of $F$, which contradicts Proposition 2.10. Hence $B_{j} \cap B_{0}=\varnothing$ for all $j$.

Q.E.D.

Proof of Theorem 2.2. By Proposition 2.8 we know already that the closure $X_{\alpha}^{*}$ of $X_{\alpha}$ is analytic for every $\alpha$. Thus it remains to show that $X_{\alpha}$ is zariski open in $X_{\alpha}^{*}$. Take the diagram obtained in Proposition 2.8 for each $\alpha$. By Lemma 2.11 $f_{\alpha}$ satisfies the condition $(\tilde{\mathrm{A}})$. Then by Lemma $2.7 Z_{0}$ for $Z=Z_{\alpha}$ and $f=f_{\alpha}$ is Zariski open in $Z_{\alpha}$. Let $A$ $=Z_{\alpha}-Z_{0}$. Recall that $\varphi_{\alpha}\left(Z_{0}\right)=X_{\alpha}$ (cf. the proof of Proposition 2.8) and $\varphi_{\alpha}\left(Z_{\alpha}\right)=X_{\alpha}^{*}$. Hence the theorem is proved if we show that $\varphi_{\alpha}(A) \cap X_{\alpha}$ $=\varnothing$. Assume the contrary and suppose that $x^{\prime}=\varphi_{\alpha}\left(z^{\prime}\right) \in X_{\alpha}$ for some $z^{\prime} \in A$. Then $F_{\alpha} \ni 0\left(x^{\prime}\right)=\varphi_{\alpha}\left(0\left(z^{\prime}\right)\right)$. Let $B^{\prime}$ be an irreducible component of the fixed point set $B$ on $Z_{\alpha}$ which contains $0\left(z^{\prime}\right)$. Since $\varphi_{\alpha}\left(B^{\prime}\right)$ 
$\cap F_{\alpha} \neq \varnothing$ and $\varphi_{\alpha}\left(B^{\prime}\right) \subseteq F, \varphi_{\alpha}\left(B^{\prime}\right) \subseteq F_{\alpha}$. Further by the definition of $Z_{0}$, $B^{\prime} \neq B_{0}$, where $B_{0}=B_{0}\left(f_{\alpha}\right)$. Then by Lemma $2.11 B^{\prime} \cap B_{0}=\varnothing$ (the condition $\tilde{\mathrm{A}} 2)$ ), and from Corollary 2.6 it follows that $B^{\prime} \neq B_{\infty}=B_{\infty}\left(f_{\alpha}\right)$. Take a point $t \in f_{\alpha}\left(B^{\prime}\right)$. Let $C_{1}, \cdots, C_{q}$ be the canonically numbered irreducible components of $C=f_{\alpha}^{-1}(t)$ and $0_{i}, \infty_{i}$ the corresponding fixed points on $C_{i}, C$ being linear by Lemma 2.5. Recall further that $C \cap B_{0}$ $=0_{1}$ and $C \cap B_{\infty}=\infty_{q}$. Since $B^{\prime} \neq B_{0}, \neq B_{\infty}, 0\left(z^{\prime}\right)=0_{k}$ for some $1<k \leqq q$. Take for each $i$ a point $z_{i} \in C_{i}-\left\{0_{i}, \infty_{i}\right\}$ and put $x_{i}=\varphi_{\alpha}\left(z_{i}\right)$. Then we get that the sequence $\left(x_{i}\right), 1 \leqq i<k$, generates a linear curve on $X$ and since $\varphi_{\alpha}\left(B_{0}\right)$ and $\varphi_{\alpha}\left(B^{\prime}\right)$ are contained in $F_{\alpha}, 0\left(x_{1}\right), \infty\left(x_{k}\right) \in F_{\alpha}$. This contradicts Proposition 2. 10. Thus $\varphi_{\alpha}(A) \cap X_{\alpha}=\varnothing$ and the proof of Theorem 2.2 is complete.

Remark 2.1. As was mentioned above, when $X$ is projective, the theorem is due to Bialynicki-Birula [1]. More generally he showed that the theorem is also true when $X$ is a complete nonsingular algebraic variety. In fact, by Sumihiro [16, Cor. 2] for every point $x \in X$ we can then take a $C^{*}$ invariant affine open neighborhood of $x$ in $X$, and hence his assumption that $X$ can be covered by $C^{*}$ invariant quasi-affine open subsets is now superfluous. As will be clear from the proof this remark applies also to Theorem 3. 3 below so that it holds true also for complete nonsingular algebraic varieties.

Remark 2.2. When $X$ is projective, Proposition 2.10 and Theorem 2.2 can also be seen as follows: First by a theorem of Blanchard (cf. $\left[12\right.$, III. 9.2]) there is an embedding $j: X \rightarrow \mathbb{P}^{n}$ such that the given $\mathbb{C}^{*}$ action is induced by a linear $\mathbb{C}^{*}$ action on $\mathbb{P}^{n}$. This reduces the problem to the case where $X=\mathbb{P}^{n}$. Hence with respect to suitable homogeneous coordinates $\left(\xi_{0}: \cdots: \xi_{n}\right)$ of $\mathbb{P}^{n}$ we may assume that the action takes the form

$$
\sigma\left(t,\left(\xi_{0}: \cdots: \xi_{n}\right)\right)=\left(t^{k_{0}} \xi_{0}: \cdots: t^{k_{n}} \xi_{n}\right) .
$$

We define the partition $\{0,1, \cdots, n\}=I_{1} \cup \cdots \cup I_{s}$, by the following condition; for any $i \in I_{\alpha}$ and $j \in I_{\beta}, k_{i}=k_{j}$ if and only if $\alpha=\beta$. We may arrange $I_{\alpha}$ in such a way that $k_{I_{1}} \leqq \cdots \leqq k_{I_{s}}$, where $k_{I_{\alpha}}=k_{i}$ for any $i \in I_{\alpha}$. 
Let $F_{\alpha}$ be the linear subspace of $\mathbb{P}^{n}$ defined by $F_{\alpha}=\left\{\left(\xi_{i}\right) ; \xi_{i}=0, i \notin I_{\alpha}\right\}$. Then $F_{\alpha}$ are the connected components of the fixed point set $F$. Let $L_{1}$ be the linear subspace of $\mathbb{P}^{n}$ defined by $L_{1}=\left\{\xi_{i}=0 ; i \in I_{1}\right\}$. Then in the B.-B. decomposition $\left\{X_{\alpha}\right\}, \alpha=1, \cdots, s$, of $\mathbb{P}^{n}$ for our $\mathbb{C}^{*}$ action, $X_{1}=\mathbb{P}^{n}$ $-L_{1}$. This implies that for every point $x \in \mathbb{P}^{n}, 0(x) \in F_{1}$ if and only if $x \notin L_{1}$. From these observations one can deduce easily the proof of our proposition and theorem by induction on $n$.

\section{$\S 3 . \quad$ Mixed Hodge Structure and Proof of Theorem}

Let $X$ be a compact Kähler manifold and $Y$ an analytic subset of $X$. Let $U$ be any Zariski open subset of $Y$. Then by Deligne [7] (cf. [10, 1.4]) for every $i \geqq 0$, $i$-th cohomology group with compact supports of $U, H_{c}^{i}(U, Q)$, has the natural mixed $Q$-Hodge structure. (For the definition of mixed Q-Hodge structures see [7,2.3].) Now suppose further that $U$ is connected and nonsingular and that $F$ is a connected submanifold of $U$ with $f: F \rightarrow U$ the inclusion. Let $D_{F}: H^{i}(F, Q) \cong H^{2 m-i}(F, Q)^{\prime}$ (resp. $D_{U}: H_{c}^{j}(U, \mathbb{Q}) \cong H^{2 n-j}(U, Q)^{\prime}$ ) be the Poincare isomorphism, where $m=\operatorname{dim} F \quad(n=\operatorname{dim} U)$ and 'denotes the dual vector space. Let $\lambda=n$ $-m$. Define the Gysin homomorphism $f_{*}: H^{i-2 \lambda}(F, Q) \rightarrow H_{c}^{i}(U, Q)$ by $f_{*}$ $=D_{U}\left(f^{*}\right)^{\prime} D_{F}$. Since $F$ is compact Kähler, the left hand side has the natural (pure) $Q$-Hodge structure of weight $i-2 \lambda$, that is, we have the natural direct sum decomposition $H^{i-2 \lambda}(F, \mathbb{Q}) \otimes \mathbb{C}=H^{i-2 \lambda}(F, \mathbb{C})=\bigoplus_{s+t=i-2 \lambda}$

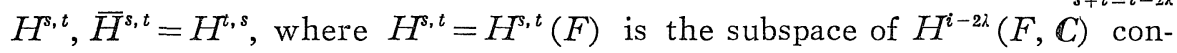
sisting of elements represented by closed $C^{\infty}$ forms of type $(s, t)$ with respect to the de Rham isomorphism (and is naturally isomorphic to $H^{t}\left(F, \Omega_{F}^{s}\right)$ ), and - denotes the complex conjugation (cf. $[7,2.2]$ ). Pure Hodge structures are regarded as special cases of mixed Hodge structures. We denote by $H^{i}(F, Q[-2 \lambda])$ the vector space $H^{i-2 \lambda}(F, Q)$ with pure Hodge structure of weight $i$ defined as follows; $H^{i}(F, Q[-2 \lambda]) \otimes C$ $=\bigoplus_{p+q=i} H^{p, q}[-2 \lambda]$ with $H^{p, q}[-2 \lambda]=H^{p-\lambda, q-\lambda}(F)$. Then as a special case of [10, Corollary 1.7.2] we have the following:

Lemma 3. 1. Suppose that $F$ is a deformation retract of $U$ so that $f_{*}$ is isomorphic. Then $f_{*}$ induces an isomorphism of mixed 
Hodge structures; $f_{*}[-2 \lambda]: H^{i}(F, \mathbb{Q}[-2 \lambda]) \cong H_{c}^{i}(U, \mathbb{Q})$. In particular the mixed Hodge structure on $H_{c}^{i}(U, \mathbb{Q})$ reduces to a pure Hodge structure of weight $i$ and we have

$$
\begin{aligned}
h_{c}^{p, q}(U) & =0 & & p+q \neq i \\
& =h^{p-\lambda, q-\lambda}(F), & & p+q=i,
\end{aligned}
$$

where $h_{c}^{p, q}(U)$ and $h^{p-\lambda, q-\lambda}(F)=\operatorname{dim} H^{p-\lambda, q-\lambda}(F)$ are the Hodge numbers of $H_{c}^{i}(U, \mathbb{Q})$ and $H^{i-2 x}(F, \mathbb{Q})$ (cf. $[7,2.3 .7]$ ).

Next, let $X$ be as above, and $Y_{0} \supseteqq Y_{1} \supseteqq \cdots \supseteqq Y_{m+1}$ with $Y_{0}=X$ and $Y_{m+1}=\varnothing$, a descending sequence of analytic subsets of $X$. Let $X_{t}=Y_{t}$ $-Y_{t+1}$. Then as mentioned above each $H_{c}^{i}\left(X_{i}, \mathbb{Q}\right)$ has the natural mixed Q-Hodge structure. We then consider the spectral sequence of Fary associated with this sequence $\left\{Y_{t}\right\}$ (cf. $[10$, Section 4] or [2, XI]);

$$
E_{2}^{s, t}=H_{c}^{s+t}\left(X_{s}, \mathbb{Q}\right) \Rightarrow H^{s+t}(X, \mathbb{Q}) .
$$

In $[10$, Proposition 4.6] we have proved in particular the following:

Lemma 3.2. The spectral sequence $(*)$ is one in (MH) (cf. $[10,4.4]$ for the definition). Hence if $(*)$ degenerates and so we have isomorphisms $H_{c}^{s+t}\left(X_{s}, \mathbb{Q}\right) \cong G r^{s} H^{s+t}(X, Q)$ for all $(s, t)$, where $G r$ denotes taking the associated graded modules. Then these isomorphisms are those of mixed $\mathbb{Q}$-Hodge structures. In particular we get that for all $p, q \geqq 0$

$$
\sum_{s=0}^{m} h_{c}^{p, q}\left(X_{s}\right)=h^{p, q}(X),
$$

where $h_{c}^{p, q}\left(X_{s}\right)$ are as in the previous lemma.

Now let $T=\left(\mathbb{C}^{*}\right)^{k}$ be an algebraic torus acting on a complex space $X$ biholomorphically. We say that this action, say, $\sigma: T \times X \rightarrow X$, meromorphic, if $\sigma$ extends to a meromorphic map $\sigma^{*}:\left(\mathbb{P}^{1}\right)^{k} \times X \rightarrow X$ with respect to the natural inclusion $\left(\mathbb{C}^{*}\right)^{k} \subseteq\left(\mathbb{P}^{1}\right)^{k}$.

Theorem 3. 3. Let $X$ be a compact Kähler manifold. Suppose that an algebraic torus $T=\left(\mathbb{C}^{*}\right)^{k}$ acts biholomorphically and mero- 
morphically on $X$ with nonempty fixed point set $F$. Let $F_{a}, 1 \leqq \alpha \leqq m$, be the connected components of $F$. Then for each $\alpha$ there is an integer $\lambda_{\alpha}$ such that

$$
h^{p, q}(X)=\sum_{\alpha=1}^{m} h^{p-\lambda_{\alpha}, q-\lambda_{\alpha}}\left(F_{\alpha}\right) .
$$

In particular $h^{p, q}(X)=0$ for $|p-q|>\operatorname{dim} F$, which is a special case of a theorem of Carrell and Lieberman [6]. Note that by Remark 1.2 we can dispense with the arguments of [6] for the proof of Corollary 1. 7 which will be used in the proof of the theorem. On the various choices of $\lambda_{\alpha}$ in the above theorem see (3.5) below.

The following lemma reduces the problem to the case of a $\mathbb{C}^{*}$ action (See also (3.5)).

Lemma 3. 4. There is an algebraic subgroup $H \subseteq T, H \cong \mathbb{C}^{*}$, whose fixed point set coincides with $F$.

Proof. Let $\Gamma \subseteq T \times X \times X$ be the graph of the given action $\sigma: T \times X$ $\rightarrow X$. Let $f: \Gamma \rightarrow X \times X$ be the natural projection and $f_{1}: \Gamma_{\Delta} \rightarrow \Delta$ the restriction of $f$ to the diagonal $\Lambda \subseteq X \times X$. Then the fiber of $f_{1}$ over $(x, x)$ $\in \Delta$ is the stabilizer $T_{x}$ of $x$ (regarding it naturally as a subgroup of $T$ ). In fact by $f_{1}, \Gamma_{\Delta}$ is a group variety over $\Delta$ with fibers $T_{x}$. Note that since the action is meromorphic, $T_{x}$ are all algebraic subgroups of $T$ (cf. [9, Lemma 2.4]). Then take a finite descending sequence $\Delta=A_{0} \supseteqq \cdots$ $\supseteqq A_{s}$ of analytic subsets of $\Delta$ such that for each $i, A_{i}-A_{i^{\perp} 1}$ is connected and the fibers of $f_{1}$ over $A_{i}-A_{i+1}$ have the same dimension so that the restriction of $f_{1}$ to the identity component of $\Gamma_{\Delta}$ over $A_{i}-A_{i+1}$ is smooth. Then since any smooth deformation of algebraic subgroups of an algebraic torus is trivial, the identity component $T_{x 0}$ of $T_{x}$ is one and the same subgroup of $T$ for all $x \in A_{i}-A_{i+1}$. This implies that there are only a finite number of subgroups, say, $H_{1}, \cdots, H_{n}$, of $T$ such that $H_{i}=T_{x 0}$ for some $x \in X$. We may suppose that $H_{1}=T$. Then we can find a one dimensional connected algebraic group $H$ of $G$ which is not contained in any of $H_{i}, i \geqq 2$. Then clearly $\operatorname{Fix}(H)=F$, and $H \cong \mathbb{C}^{*}$. Q.D.

Before proving the theorem we give the following remark. Let 
$\left\{X_{\alpha}\right\}$ be any meromorphic decomposition of a compact complex space $X$. Then since $X_{\alpha}$ are irreducible by definition, we can always find a descending sequence $X=Y_{0} \supseteqq Y_{1} \supseteqq \cdots \supseteqq Y_{r} \cdots$ of analytic subsets of $X$ such that $Y_{t}-Y_{t+1}=X_{\alpha}$ for a unique $\alpha=\alpha(t)$. We call any such sequence $\left\{Y_{t}\right\}$ associated with $\left\{X_{\alpha}\right\}$ though such a sequence is not unique.

Proof of Theorem 3. 3. By the above lemma we have only to consider the case where $k=1$. Namely we take a surjective rational homomorphism $h: \mathbb{C}^{*} \rightarrow H$ with $H$ as in the lemma and then take the induced $\mathbb{C}^{*}$ action. Let $\left\{X_{a}\right\}$ be the B.-B. decomposition of $X$ with respect to this $\mathbb{C}^{*}$ action. Take and fix a descending sequence $\left\{Y_{t}\right\}$ of $X$ associated with $\left\{X_{\alpha}\right\}$, which makes sense because $\left\{X_{\alpha}\right\}$ are meromorphic by Theorem 2.2. Thus $Y_{t}-Y_{t+1}=X_{\alpha}$ for a unique $\alpha=\alpha(t)$. For convenience we shall write $X_{t}$ instead of $X_{\alpha(t)}, 0 \leqq t \leqq m-1$. Consider now the spectral sequence $(*)$ of Fary associated with $\left\{Y_{t}\right\}$. Then we have the inequality

$$
\sum_{t} \operatorname{dim} E_{2}^{i-t, i}=\sum_{t} \operatorname{dim} H_{c}^{i}\left(X_{t}, Q\right) \geqq \operatorname{dim} H^{i}(X, Q), i \geqq 0 .
$$

On the other hand, since $F_{t}$ is a deformation retract of $X_{t}$ by Lemma 2.1, we have the isomorphisms $f_{t^{*}}: H^{i-\lambda_{t}}\left(F_{t}, Q\right) \cong H^{i}\left(X_{t}, \mathbb{Q}\right)$, where $f_{t}$ : $F_{t} \rightarrow X$ are the inclusions and $\lambda_{t}$ are as in Lemma 2.1. Hence we get that for $a=0,1$

$$
\sum_{i \equiv a(2)} \operatorname{dim} H^{i}(F, \mathbb{Q})=\sum_{i \equiv a(2)} \sum_{i} \operatorname{dim} H^{i}\left(F_{t}, \mathbb{Q}\right) \geqq \sum_{i \equiv a(2)} \operatorname{dim} H^{i}(X, \mathbb{Q}) .
$$

Combining this with the inequality of Conner (Corollary 1.7 for the vector field generating the $\mathbb{C}^{*}$ action) we see at once that this must be an equality, and hence so must be (2). Thus the spectral sequence $(*)$ degenerates. Then by Lemmas 3.1 and 3.2 we get that

$$
h^{p, q}(X)=\sum_{t} h_{c}^{p, q}\left(X_{t}\right)=\sum_{t} h^{p-\lambda_{t}, q-\lambda_{t}}\left(F_{t}\right)
$$

for all $(p, q)$.

In Theorem 3. 3 the integers $\lambda_{\alpha}$ are determined each time we take an algebraic 1-parameter subgroup of $T$ i.e. a rational homomorphism $h: \mathbb{C}^{*}$ $\rightarrow T$. We shall state this dependence of $\lambda_{\alpha}$ on $h$ more explicitly. We need some notation. 
Let $T$ be in general an algebraic torus (over $\mathbb{C}$ ) of dimension $k>0$. Let $\mathfrak{X}$ (resp. $\mathfrak{P}$ ) be the set of rational characters (resp. algebraic 1parameter subgroups) of $T$. They have the natural structure of free $\mathbb{Z}$ modules of rank $k$ and there is a natural bilinear pairing $\langle\rangle:, \mathfrak{X} \times \mathfrak{F}$ $\rightarrow \mathbb{Z}$; if we fix an isomorphism $T \cong \mathbb{C}^{*}\left(t_{1}\right) \times \cdots \times \mathbb{C}^{*}\left(t_{k}\right)$, then $\chi \in \mathbb{X}$ (resp. $h \in \mathfrak{P})$ corresponds to $\left(m_{1}, \cdots, m_{k}\right) \in \mathbb{Z}^{k}$ if and only if $\chi\left(t_{1}, \cdots, t_{k}\right)=t_{1}^{m_{1}} \cdots$ $t_{k}^{m_{k}}$ (resp. $\left.h(t)=\left(t^{m_{1}}, \cdots, t^{m_{k}}\right), \mathbb{C}^{*}=\mathbb{C}^{*}(t)\right)$, and for any $\chi \in \mathfrak{X}$ and $h \in \mathfrak{P}$, $\langle\chi, h\rangle=m$ iff $\chi \cdot h(t)=t^{m}$. $\langle$,$\rangle extends naturally to a \mathbb{R}$-bilinear paring $\langle,\rangle_{\boldsymbol{R}}: \mathfrak{X}_{\boldsymbol{R}} \times \mathfrak{P}_{\boldsymbol{R}} \rightarrow \mathbb{R}$, where $\mathfrak{r}_{\boldsymbol{R}}=\mathfrak{i} \otimes_{\boldsymbol{Z}} \mathbb{R}, \mathfrak{P}_{\boldsymbol{R}}=\mathfrak{P}_{\boldsymbol{Z}} \otimes_{\boldsymbol{Z}}$.

Let $\rho: T \rightarrow G L(V)$ be any rational representation of $T$ on a finite dimensional vector space $V$. Let $\mathscr{A}^{\rho}=\left\{\chi_{1}, \cdots, \chi_{n}\right\}$ be the set of rational characters of $T$ obtained by diagonalizing $\rho$ (possibly $\chi_{i}=\chi_{i}, i \neq j$ ), where $n=\operatorname{dim} V$. We assume that $\chi_{j} \neq 0$ for any $j$. Let $H_{x_{i}}$ be the hyperplane in $\mathfrak{P}_{\boldsymbol{R}}$ defined by $H_{x_{i}}=\left\{h \in \mathfrak{P}_{\boldsymbol{R}} ;\left\langle\chi_{i}, h\right\rangle_{\boldsymbol{R}}=0\right\}$. Further we define a function $\lambda=\lambda^{\circ}$ on $\mathfrak{P}_{\boldsymbol{R}}$ by $\lambda(h)=\#\left\{i ; \chi_{i}(h)>0\right\}$. Clearly $0 \leqq \lambda \leqq n$ and $\lambda$ is constant on each connected component of $\mathfrak{P}_{\mathbb{R}}-\bigcup_{i} H_{\chi_{i}}$.

Now let $X, T, F$ and $F_{\alpha}$ be as in Theorem 3.3. Take any point $x_{\alpha}$ from each $F_{\alpha}$, and let $\rho_{\alpha}: T \rightarrow G L\left(V_{a}\right)$ be the associated rational representation (cf. [9, Proposition 2.7]), where $V_{\alpha}$ is the fiber over $x_{\alpha}$ of the normal bundle $N_{F_{\alpha^{\prime}} X}$ of $F_{\alpha}$ in $X$. Then as above we have for each $F_{\alpha}$ the set of characters $\left\{\chi_{1}^{\alpha}, \cdots, \chi_{r_{\alpha}}^{a}\right\}, r_{\alpha}=\operatorname{codim}\left(F_{\alpha}, X\right)$, hyperplanes $H_{\chi_{i}^{\alpha}}$ in $\mathfrak{P}_{\boldsymbol{R}}$ corresponding to them and the functions $\lambda^{\alpha}=\lambda^{\rho_{\alpha}}$ on $\mathfrak{P}_{\boldsymbol{R}}$. (These are independent of the choices of $x_{\alpha}$ ) One then sees readily the following:

(3.5) 1) The induced $\mathbb{C}^{*}$ action on $X$ by an element $h \in \mathfrak{P}$ has the same fixed point set $F$ as $T$ if and only if $h \notin H_{\chi_{i}^{\alpha}}$ for any $\chi_{i}^{\alpha}$. (Call such an $h$ regular.) 2) For regular $h, \lambda_{\alpha}$ in Theorem 3.3 are given by $\lambda_{\alpha}=\lambda^{\alpha}(h)$. In particular for each connected component of the set $\mathfrak{P}_{\boldsymbol{R}}-\cup_{i, \alpha} H_{\chi_{i}^{\alpha}}$ there is one choice (and hence in all a finite number of choices) of the set of integers $\left\{\lambda_{a}\right\}$ as in Theorem 3. 3.

More generally we see that for all $h$ in one and the same connected component of $\mathfrak{P}_{\mathbb{R}}-\cup H_{\chi_{i}^{\alpha}}$, the B.-B. decompositions induced by the corresponding $C^{*}$ actions are the same, as analytic decompositions. Therefore we get the following: 
(3.6) Associated with each connected component of $\mathfrak{P}_{\mathbb{R}}-\cup_{i, \alpha} H_{\chi_{i}^{\alpha}}$ we have naturally a meromorphic decomposition $\left\{X_{\alpha}\right\}$ of $X$.

On the other hand, note that $\mathfrak{P}_{\boldsymbol{C}}=\mathfrak{P} \otimes_{\boldsymbol{Z}} \boldsymbol{C}$ can naturally be considered as the vector space of analytic 1-parameter subgroups of $T$ i.e. complex analytic homomorphism $h: C \rightarrow T$, and hence as the Lie algebra of $T$. Take the maximal real subtorus $T_{\mathbb{R}}$ of $T$ so that $T$ is the complexification of $T_{\boldsymbol{R}}$ and that we have the natural isomorphism $\varphi: \mathrm{t}_{\boldsymbol{R}} \otimes_{\mathbb{R}} \boldsymbol{C} \cong \mathfrak{P}_{\boldsymbol{C}}, \mathrm{t}_{\boldsymbol{R}}$ corresponding to $\mathfrak{P}_{\boldsymbol{R}}$, where $\mathrm{t}_{\boldsymbol{R}}$ is the (real) Lie algebra of $T_{\boldsymbol{R}}$. If the action of $T$ on $X$ is effective, then $t_{\mathbb{R}}$ can further be considered as a subspace of Killing vector fields on $X$ with respect to a suitable Kähler metric on $X$. Thus to any such Killing vector field $V$ on $X$ we can associate integers $\lambda_{a}(V)$ by $\lambda_{\alpha}(V)=\lambda^{\alpha}(\varphi(V))$. Note that if $V$ generates $\mathrm{t}_{\boldsymbol{R}}$, then $\varphi(V)$ is regular in the sense that $\varphi(V) \notin H_{\chi_{i}^{\alpha}}$ for any $\chi_{i}^{\alpha}$ as above.

Now we come to the proof of Theorem stated in the introduction.

Proof of Theorem. By Lemma 1.4 and Theorem 3.3, up to the choices of $\lambda_{\alpha}$ it is enough to show that every biholomorphic action of $\left(\mathbb{C}^{*}\right)^{k}$ on $X$ is meromorphic if $F \neq \varnothing$. Indeed, this is a special case of a theorem of Sommese [15] (cf. also [9, Proposition 6. 10]). As for $\lambda_{\alpha}$, assuming that the action is effective as we may, by the above remark we may take $\lambda_{\alpha}=\lambda_{\alpha}(V)$ by taking $h \in \Re$ in the above proof from the connected component to which $\varphi(V)$ belongs. One sees readily that these $\lambda_{\alpha}$ coincide with those used by Frankel in [8] (see also [12, III. 10]).

Q.E.D.

We end this paper with the following more or less wellknown:

Example. Let $G$ be a connected semisimple algebraic group (over C), $B$ a Borel subgroup of $G$ and $T$ a maximal torus of $G$ contained in $B$. Let $T$ act on the homogeneous space $X=G / B$ on the left and $F$ be the set of fixed points of this action. Let $N(T)$ be the normalizer of $T$ in $G$ and $W=N(T) / T$ the Weyl group of $G$ with respect to $T$. Fix a representative $\left\{w_{\alpha}\right\}_{\alpha=1, \ldots, m}, w_{1}=e$, in $N(T)$ of elements of $W$, where 
$m=\# W$. Then we get that $F=\left\{x_{\alpha}=w_{\alpha} B \in X ; 1 \leqq \alpha \leqq m\right\} \quad$ (cf. [4, p. 278]). In particular $F$ consists of isolated points and $\# F=m$. Hence by Theorem $3.3 h^{p, q}(X)=0, p \neq q$, as is wellknown. Moreover fixing a regular 1-parameter subgroup $h \in \mathfrak{P}$ as in (3.5) we have an integer $\lambda_{\alpha}$ $=\lambda^{\alpha}(h) \geqq 0$ for each $\alpha$ with $h^{p, p}(X)=\#\left\{\alpha ; \lambda_{\alpha}=p\right\}$ (cf. [6, Remark 4)]). We shall now interprete the right hand side in terms of the Weyl group. Let $\rho_{\alpha}: T \rightarrow G L\left(V_{\alpha}\right)$ be the rational representation as in (3.5), where $V_{\alpha}$ is now the tangent space of $X$ at $x_{\alpha}$. Then we see readily that via the natural isomorphism $w_{\alpha^{*}}: V_{1} \cong V_{\alpha}, \rho_{\alpha}$ is equivalent to $\rho_{w_{\alpha}}=\rho \cdot \widetilde{w}_{\alpha}: T \rightarrow$ $G L\left(V_{1}\right)$ where $\widetilde{w}_{\alpha}$ is the automorphism of $T$ induced by $w_{\alpha}$. Let $\left\{\chi_{1}^{\alpha}, \cdots\right.$, $\left.\chi_{n}^{\alpha}\right\}$ be the set of characters of $\rho_{\alpha}$, or of $\rho_{w_{\alpha}}$, where $n=\operatorname{dim} X$. We shall write $\chi_{i}$ for $\chi_{i}^{1}$. Since the Weyl group induces permutations of the set $\left\{\chi_{1}, \cdots, \chi_{n},-\chi_{1}, \cdots,-\chi_{n}\right\}$ with respect to the natural induced action on $X$, we get that the characters of $o_{w_{\alpha}}$ are of the form $\left\{\varepsilon_{1}^{\alpha} \chi_{1}, \cdots, \varepsilon_{n}^{\alpha} \chi_{n} ; \varepsilon_{i}^{\alpha}\right.$ $= \pm 1\}$. In particular $\mathfrak{P}_{\boldsymbol{R}}-\cup_{i, \alpha} H_{x_{i}^{\alpha}}=\mathfrak{P}_{\boldsymbol{R}}-\bigcup_{i} H_{x_{i}}$. Let $U$ be the connected component of $\mathfrak{P}-\cup H_{x_{i}}$ such that $\chi_{i}(h)>0$ for all $i$ and $h \in U$ (positive Weyl chamber). Then by the definition of the function $\lambda^{\alpha}(h)$ if we take $h$ from $U$, we have that $\lambda^{\alpha}(h)=s\left(w_{\alpha}\right)$, where $s\left(w_{\alpha}\right)=\#\left\{i ; \varepsilon_{i}^{\alpha}=-1\right\}$. Thus we have the following formula:

$$
h^{p, p}(X)=\left\{\alpha ; s\left(w_{\alpha}\right)=p\right\} .
$$

The result is classical and due to Chevalley, Bott, and Borel and Hirzebruch (cf. [5, 24.4]. See also [8, p. 7]).

On the other hand, by (3.6) for each connected component of $\mathfrak{P}_{\boldsymbol{R}}$ $-\cup_{i} H_{x_{i}}$ (Weyl chamber) there corresponds a unique meromorphic (or algebraic) decomposition $\left\{X_{\alpha}\right\}$ of $X$, and they are transformed to each other by the elements of $W$. Since $F$ is isolated, by [1] (cf. Lemma 2.1) $X_{\alpha} \cong \mathbb{C}^{\lambda_{\alpha}}$ for each $\alpha$ and hence $\left\{X_{\alpha}\right\}$ defines an analytic cellular decomposition of $X$ in the sense of [3]. In fact one can show that this decomposition $\left\{X_{\alpha}\right\}$ (corresponding to $-U$ ) coincides with the one originally constructed by Borel in [3] (see also [4, p. 347]) using the $B$-orbits (Schubert cycles). Note that the above formula $(*)$ also follows directly from this decomposition because we have then that $h^{p, p}(X)=\#\left\{\alpha: \lambda_{\alpha}\right.$ $\left.=\operatorname{dim} X_{\alpha}=p\right\}$. 


\section{References}

[1] Bialynicki-Birula, A., Some theorems on actions of algebraic groups, Ann. of Math., 98 (1973), 480-497.

[2] Borel, A., Seminar on transformation groups, Ann. of Math. Studies, 46, 1960.

[3] Kählerian coset spaces of semi-simple Lie groups, Proc. Nat. Acad. Sci. $U S A, 40$ (1954), 1147-1151.

[4] Borel, A., Linear algebraic groups, Benjamin, New York, 1969.

[5] Borel, A. and Hirzebruch, F., Characteristic classes and homogeneous spaces II, Amer. J. Math., 81 (1959), 315-382.

[6] Carrell, J. B. and Lieberman, D. I., Holomorphic vector fields on compact Kähler manifolds, Invent. Math., 21 (1973), 303-309.

[7] Deligne, P., Théories de Hodge II, III, Publ. Math. IHES, 40 (1972), 5-57 and 44 (1975), 5-77.

[8] Frankel, T. T., Fixed points and torsions on Kähler manifolds, Ann. of Math., 70 (1959), 1-8.

[9] Fujiki, A., On automorphism groups of compact Kähler manifolds, Invent. Math., 44 (1978), 225-258.

[10] - Duality of mixed Hodge structures of algebraic varieties, to appear in Publ. RIMS, Kyoto Univ.

[11] Kaup, W., Reele Transformations Gruppen und invariante Metriken auf komplexen Räumen. Invent. Math., 3 (1967), 43-70.

[12] Kobayashi, S., Transformation groups in differential geometry, Springer, BerlinHeidelberg-New York, 1972.

[13] Kosniowski, C., Applications of holomorphic Lefschetz formula, Bull. London Math. Soc., 2 (1970), 43-48.

[14] de Rham, G., Sur la division de formes et de courants par une forme linéaire, Comment. Math. Helv., 28 (1954), 346-352.

[15] Sommese, A. J., Extension theorems for reductive group actions on compact Kähler manifolds, Math. Ann. 218 (1975), 107-116.

[16] Sumihiro, H., Equivariant completion, Journal of Math. Kyoto Univ. 14 (1974), $1-28$.

Note added in proof. After submitting the paper the author was informed that the same resluts had also been obtained by J. B. Carrell and A. J. Sommese independently in the following two articles.

[17] Carrell, J. B. and Sommese, J. B. C* actions, Math. Scand. 43 (1978), 49-60.

[18] Carrell, J. B. and Sommese, J. B., Some topological aspects of $C^{*}$ actions on compact Kähler manifolds, preprint. 